\title{
AN ATLAS OF GALAXY SPECTRAL ENERGY DISTRIBUTIONS FROM THE ULTRAVIOLET TO THE MID-INFRARED
}

\author{
Michael J. I. Brown ${ }^{1,2,13}$, John Moustakas ${ }^{3}$, J.-D. T. Smith ${ }^{4}$, Elisabete da Cunha ${ }^{5}$, T. H. Jarrett ${ }^{6}$, \\ Masatoshi Imanishi $^{7,8,9}$, Lee Armus $^{10}$, Bernhard R. Brandi ${ }^{11}$, And J. E. G. Peek ${ }^{12,14}$ \\ ${ }^{1}$ School of Physics, Monash University, Clayton, Victoria 3800, Australia \\ ${ }^{2}$ Monash Centre for Astrophysics, Monash University, Clayton, Victoria 3800, Australia \\ ${ }^{3}$ Department of Physics and Astronomy, Siena College, 515 Loudon Road, Loudonville, NY 12211, USA \\ ${ }^{4}$ Department of Physics and Astronomy, University of Toledo, Ritter Observatory, MS \#113, Toledo, OH 43606, USA \\ ${ }^{5}$ Max-Planck-Institut für Astronomie, Kšnigstuhl 17, D-69117 Heidelberg, Germany \\ ${ }^{6}$ Department of Astronomy, University of Cape Town, Private Bag X3, Rondebosch 7701, South Africa \\ ${ }^{7}$ Subaru Telescope, 650 North A'ohoku Place, Hilo, HI 96720, USA \\ ${ }^{8}$ Department of Astronomy, School of Science, Graduate University for Advanced Studies (SOKENDAI), Mitaka, Tokyo 181-8588, Japan \\ ${ }^{9}$ National Astronomical Observatory of Japan, 2-21-1 Osawa, Mitaka, Tokyo 181-8588, Japan \\ ${ }^{10}$ Spitzer Science Center, California Institute of Technology, Pasadena, CA 91125, USA \\ ${ }^{11}$ Leiden Observatory, Leiden University, P.O. Box 9513, 2300-RA Leiden, The Netherlands \\ ${ }^{12}$ Department of Astronomy, Columbia University, New York, NY 10027, USA \\ Received 2013 December 10; accepted 2014 February 21; published 2014 May 15
}

\begin{abstract}
We present an atlas of 129 spectral energy distributions for nearby galaxies, with wavelength coverage spanning from the ultraviolet to the mid-infrared. Our atlas spans a broad range of galaxy types, including ellipticals, spirals, merging galaxies, blue compact dwarfs, and luminous infrared galaxies. We have combined ground-based optical drift-scan spectrophotometry with infrared spectroscopy from Spitzer and Akari with gaps in spectral coverage being filled using Multi-wavelength Analysis of Galaxy Physical Properties spectral energy distribution models. The spectroscopy and models were normalized, constrained, and verified with matched-aperture photometry measured from Swift, Galaxy Evolution Explorer, Sloan Digital Sky Survey, Two Micron All Sky Survey, Spitzer, and Wide-field Infrared Space Explorer images. The availability of 26 photometric bands allowed us to identify and mitigate systematic errors present in the data. Comparison of our spectral energy distributions with other template libraries and the observed colors of galaxies indicates that we have smaller systematic errors than existing atlases, while spanning a broader range of galaxy types. Relative to the prior literature, our atlas will provide improved $K$-corrections, photometric redshifts, and star-formation rate calibrations.
\end{abstract}

Key words: atlases - galaxies: general - galaxies: photometry - techniques: spectroscopic

Online-only material: color figures, figure set, machine-readable tables

\section{INTRODUCTION}

Templates and models of galaxy spectral energy distributions (SEDs) are often essential for deriving the physical properties of galaxies from observables. $K$-corrections, where rest-frame colors and luminosities are derived from observed galaxy photometry, rely on galaxy SED templates and models (e.g., Oke \& Sandage 1968; Pence 1976; Coleman et al. 1980). Photometric redshifts rely on accurate models of the relationship between observed galaxy photometry and redshift. While this relationship can be modeled empirically for some galaxies using polynomial fits and neural networks (e.g., Connolly et al. 1995; Firth et al. 2003), models and templates are required for photometric redshifts of faint and high redshift galaxies.

The most extensively used galaxy SED template libraries are those of Coleman et al. (1980) and Kinney et al. (1996). These SEDs have proved exceptionally useful over several decades, but have understandable limitations. The wavelength coverage of Coleman et al. (1980) and Kinney et al. (1996) templates is limited to the ultraviolet and optical, so they are often extended into the infrared with stellar population synthesis models (e.g., Bruzual \& Charlot 2003). The Coleman et al. (1980) and Kinney et al. (1996) spectra are often of galaxy nuclei, which may not

\footnotetext{
${ }^{13}$ ARC Future Fellow.

${ }^{14}$ Hubble Fellow.
}

be representative of integrated galaxy spectra. These templates also predate the availability of precisely calibrated wide-field imaging, which can be used to normalize and verify SEDs. Without such imaging, there is the potential for systematic error, especially when combining spectra from different instruments using different extraction apertures.

With the advent of imaging and spectroscopy from the Infrared Space Observatory (Kessler et al. 1996), Spitzer Space Telescope (Werner et al. 2004), Akari (Astro-F; Murakami et al. 2007), and the Wide-field Infrared Space Explorer (WISE; Wright et al. 2010), it became both important and possible to extend galaxy SED templates into the mid-infrared (e.g., Chary \& Elbaz 2001; Polletta et al. 2007; Rieke et al. 2009). These templates include emission from dust, silicate absorption, and features attributed to polycyclic aromatic hydrocarbons (PAHs), which dominate the mid-infrared spectra of star-forming galaxies. However, large aperture spectrophotometry over a broad wavelength range remained unavailable (or unutilized), and consequently many of these templates rely upon model galaxy spectra rather than observed galaxy spectra.

Galaxy SEDs can be modeled using models of stellar populations, nebular emission lines, dust obscuration, and dust emission (e.g., Tinsley 1968; Silva et al. 1998; Charlot \& Longhetti 2001; Bruzual \& Charlot 2003; Maraston 2005; da Cunha et al. 2008; Vega et al. 2008; Pacifici et al. 2012). The models have improved considerably over the decades, with advances in 

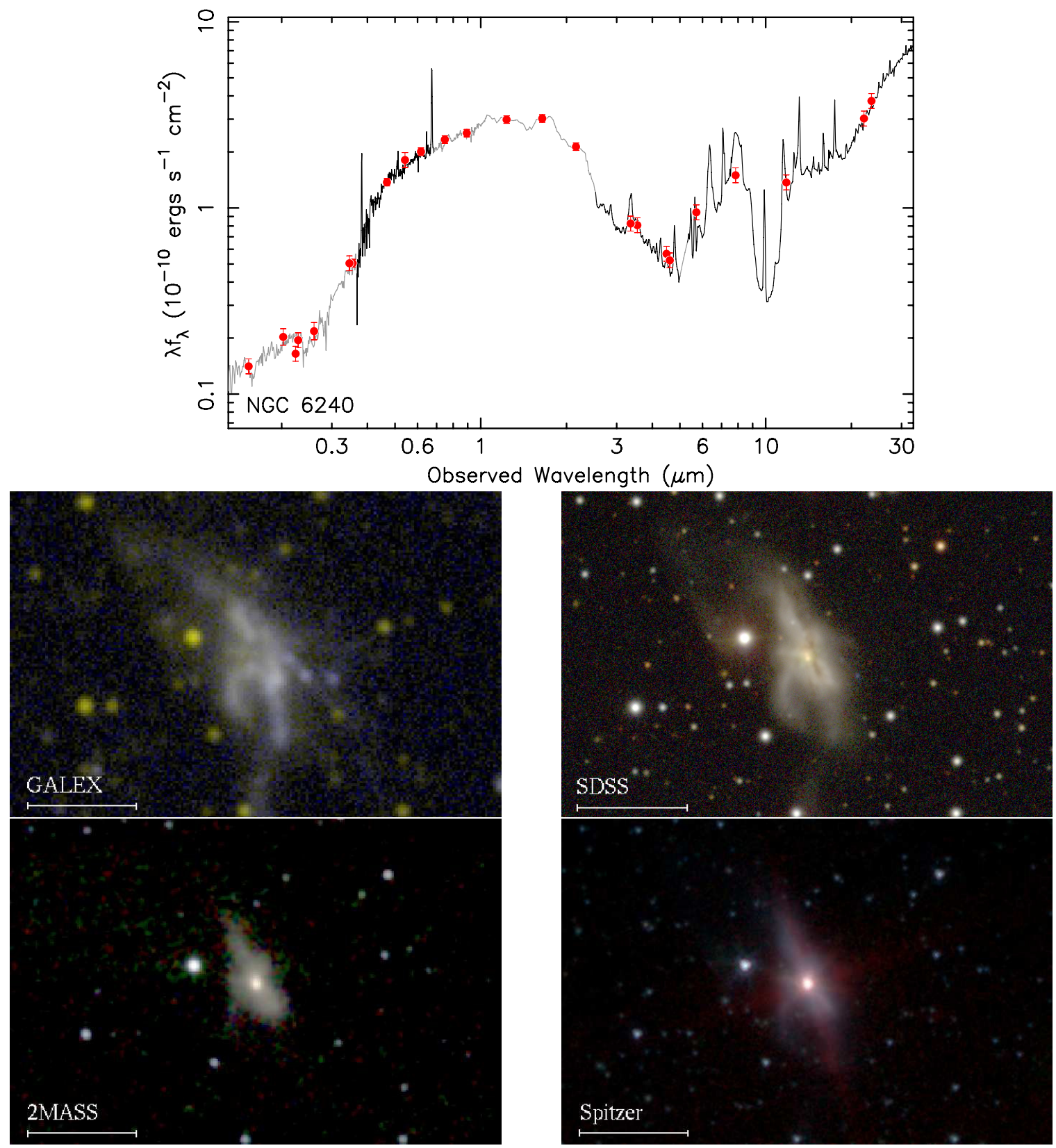

Figure 1. Ultraviolet to mid-infrared SED of NGC 6240 (top panel), along with some of the GALEX, SDSS, 2MASS, and Spitzer images that were used to constrain and verify the SED. The horizontal bar denotes an angular scale of $1^{\prime}$. In the top panel, the observed and model spectra are shown in black and gray respectively, while the photometry used to constrain and verify the spectra is shown with red dots.

(A color version of this figure is available in the online journal.)

relevant theory and stellar libraries, combined with better constraints from observed galaxy spectra and photometry. When provided with sufficiently precise photometry with broad wavelength coverage, galaxy SED models are able to do a remarkably good job of reproducing the spectra of some galaxies (see Section 3).

Unfortunately, SED models with a large number of free parameters cannot be expected to reproduce the SED of a galaxy with a complex star formation history when only limited photometry is available (e.g., Pforr et al. 2012). Similarly, SED models are not always the best means of predicting the observed colors of galaxies as a function of redshift, as models can generate unrealistic spectra. These issues also apply (albeit to a lesser extent) to modeling SEDs with combinations of empirical component spectra (e.g., Blanton \& Roweis 2007; Assef et al. 2008). This being the case, there is still a role for galaxy SED templates derived from real galaxies.

To address this need, we present an atlas of 129 galaxy SEDs with wavelength coverage spanning from the ultraviolet to the mid-infrared. Relative to the prior literature, our atlas spans a broader range of absolute magnitudes $\left(-14.7>M_{g}>-23.2\right)$, colors $(0.1<u-g<1.9)$ and galaxy types, including ellipticals, spirals, merging galaxies, blue compact dwarfs, and luminous infrared galaxies. Our templates make use of optical drift-scan spectroscopy from ground-based telescopes and midinfrared spectroscopy from Spitzer and Akari, and these spectra were scaled and verified using matched-aperture photometry in 26 passbands. In Figure 1 we present one of the SEDs along 
with color composite images of the Galaxy Evolution Explorer (GALEX), Sloan Digital Sky Survey (SDSS), and Spitzer imaging used to constrain and verify the SED.

The structure of our paper is as follows. In Section 2 we describe the galaxy sample, spectra, and photometry (all photometry presented in this paper uses the $\mathrm{AB}$ magnitude system). In Section 3 we describe how we compiled the SEDs, including filling gaps in spectral coverage with models and power laws. We compare our spectra against previous template libraries and the observed galaxy colors in Section 4, and summarize our major conclusions in Section 5. In Appendix A we introduce an illustrative subsample of 18 galaxies, and in Appendix B we present SEDs and optical images for the entire sample, all of which are available online. ${ }^{15}$

\section{DATA}

Our sample consists of $z<0.05$ galaxies with archival driftscan optical spectroscopy, all of which have well calibrated multi-wavelength imaging spanning from the ultraviolet to the mid-infrared. Almost all galaxies in our sample have spectra from the Spitzer Space Telescope's Infrared Spectrograph (IRS; Houck et al. 2004), with the exceptions being elliptical galaxies with (approximately) Rayleigh-Jeans spectra in the mid-infrared. The bulk of the optical spectra are taken from Moustakas \& Kennicutt (2006) and Moustakas et al. (2010), who used the Bok $2.3 \mathrm{~m}$ and CTIO $1.5 \mathrm{~m}$ telescopes, with some additional spectra of elliptical galaxies taken from Kennicutt (1992) and Gavazzi et al. (2004). Akari Infrared Camera (IRC; Onaka et al. 2007) spectroscopy is used when it captures a significant fraction of the total galaxy flux and has high signal to noise. Accurate matched-aperture photometry from the ultraviolet to the mid-infrared was measured with archival imaging from the GALEX (Morrissey et al. 2007), Swift UV/optical monitor telescope (UVOT; Roming et al. 2005), SDSS III (Aihara et al. 2011), Two Micron All Sky Survey (2MASS; Skrutskie et al. 2006), Spitzer Space Telescope (Fazio et al. 2004; Rieke et al. 2004), and WISE (Wright et al. 2010).

Sample selection was set by the availability of suitable spectra and images, which fortunately results in a very diverse (but not necessarily representative) sample of 129 nearby galaxies. Our requirement for multi-wavelength images and spectroscopy results in our sample (largely) being a subset of other surveys of the nearby universe. For example, our sample size is smaller than that of Moustakas \& Kennicutt (2006), who presented optical drift-scan spectroscopy of 417 galaxies, and our sample size is smaller than the 202 LIRGs and ULIRGs studied by the Great Observatories All-sky LIRG Survey (GOALS; Armus et al. 2009). Readers may find that the utility of our SED atlas is increased by combining it with detailed studies of nearby galaxies (many of which use data that we have now incorporated into this paper), including measurements of star formation rates (e.g., Moustakas et al. 2006; Kennicutt et al. 2009), fitting or modeling the mid-infrared spectra (e.g., Smith et al. 2007b; Inami et al. 2013; Stierwalt et al. 2013), and estimates of the contribution of active galactic nuclei (AGNs) to mid-infrared emission (Petric et al. 2011). In Table 1 we summarize the basic properties of our sample, including names, coordinates, morphologies, magnitudes, and redshifts.

\footnotetext{
15 The SEDs, along with images used to constrain and verify the SEDs, are available via http://dx.doi.org/10.5072/03/529D3551F0117.
}

\subsection{Photometry}

Throughout this paper we use AB apparent magnitudes that are defined by

$m=-2.5 \log \left[\left(\int R(v) \frac{f_{v}(v)}{h v} d v\right) \times\left(\int R(v) \frac{g_{v}(v)}{h v} d v\right)^{-1}\right]$

(e.g., Hogg et al. 2002), where $f_{v}$ is the SED of the source, $g_{v}$ is the SED of a source that is $3631 \mathrm{Jy}$ at all wavelengths (i.e., $m=0), R_{v}$ is the filter response function (defined as electrons per incident photon), and $h v$ is the energy of a photon with frequency $v$. Please note that these magnitudes are based on photon counts rather than fluxes, and do not simply correspond to monochromatic flux densities at the effective wavelengths of the relevant filters.

The 2MASS and Multiband Imaging Photometry for SIRTF (MIPS) filter curves (available from their respective archives) are defined as electrons per $f_{\lambda}$ (then renormalized to peak at 1.00), so we modified these curves by dividing the filter curves by $\lambda$. The MIPS zero-points provided with the archival imaging are defined using a flux density at a specific wavelength and a spectrum where the flux density varies with wavelength (i.e., a Rayleigh-Jeans spectrum). For these data, we applied corrections to the zero-points so the resulting photometry is defined by Equation (1). Photometry originally defined using the Vega magnitude system (e.g., 2MASS) has been shifted into the AB magnitude system. ${ }^{16}$ The SDSS calibration is not quite on the $\mathrm{AB}$ magnitude system, so we added corrections of -0.04 and +0.02 mag to the $u$ and $z$ photometry, respectively, to shift it back to the AB system. ${ }^{17}$

Foreground dust extinction was modeled using the Planck dust extinction maps (Planck Collaboration et al. 2011, 2013). We use the Fitzpatrick (1999) model of dust extinction as a function of wavelength, with a modified parameterization for $c_{4}$ (Peek 2013),

$$
c_{4}=4.64-11.64 \times E(B-V) .
$$

This accounts for the enhanced ultraviolet extinction observed at high Galactic latitudes (Peek \& Schiminovich 2013) relative to the expectation of the Fitzpatrick (1999) extinction curve.

Accurate matched-aperture photometry was essential for normalizing and verifying our spectra, and for constraining models used to fill gaps in our spectral coverage. Where possible, we made use of archival imaging that is readily available online. Ultraviolet imaging was taken from GALEX Release 6 (GR6) and the Swift UVOT (Roming et al. 2005), with the latter often requiring stacking of individual exposures using Swarp (Bertin et al. 2002). While some galaxies have ultraviolet imaging from the XMM-Newton optical monitor (Mason et al. 2001), these images often suffer from scattered light and were not used in the analyses presented here. The only sample galaxy without GALEX or Swift imaging is UGCA 410 (a blue compact dwarf with weak PAH emission) where we used $20^{\prime \prime} \times 10^{\prime \prime}$ elliptical aperture fluxes from the International Ultraviolet Explorer (Kinney et al. 1993) to measure an approximate total magnitude.

All galaxies in the sample have optical imaging from SDSS III (Aihara et al. 2011), near-infrared imaging from 2MASS

\footnotetext{
16 We assume the AB magnitudes of Vega are $J=0.89, H=1.37$, and $K_{S}=1.84$.

17 http://www.sdss3.org/dr8/algorithms/fluxcal.php\#SDSStoAB
} 
Table 1

Basic Properties of the Sample

\begin{tabular}{|c|c|c|c|c|c|c|c|c|c|c|}
\hline Name & J2000 Coordinates & Morphology & T-type & $\begin{array}{l}\text { BPT } \\
\text { Class }\end{array}$ & $\begin{array}{c}E(B-V) \\
(\mathrm{mag})\end{array}$ & $\begin{array}{c}g^{\mathrm{a}} \\
(\mathrm{mag})\end{array}$ & $\begin{array}{c}u-g \\
(\mathrm{mag})\end{array}$ & $\begin{array}{c}M_{g}{ }^{\mathrm{a}} \\
(\mathrm{mag})\end{array}$ & $\begin{array}{c}c z \\
\left(\mathrm{~km} \mathrm{~s}^{-1}\right)\end{array}$ & $\begin{array}{c}D_{L} \\
(\mathrm{Mpc})\end{array}$ \\
\hline $\operatorname{Arp} 256 \mathrm{~N}$ & $001850.2-102144$ & $\mathrm{SB}(\mathrm{s}) \mathrm{c}$ & 4.5 & SF & 0.03 & 14.32 & 0.84 & -20.96 & 8184 & 114.3 \\
\hline Arp $256 \mathrm{~S}$ & $001850.9-102239$ & $\mathrm{SB}(\mathrm{s}) \mathrm{b}$ & 3.0 & SF & 0.03 & 14.36 & 0.75 & -20.90 & 8124 & 113.4 \\
\hline NGC 0337 & $005950.0-073435$ & SBd & 7.0 & SF & 0.10 & 11.83 & 0.91 & -19.91 & 1648 & 22.4 \\
\hline CGCG 436-030 & $012002.6+142143$ & Pec & $\cdots$ & SF & 0.06 & 14.58 & 1.00 & -20.98 & 9353 & 129.9 \\
\hline NGC 0474 & $012006.7+032456$ & $(\mathrm{R}) \mathrm{SA}(\mathrm{s}) 0$ & -2.0 & $\cdots$ & 0.04 & 11.78 & 1.61 & -20.78 & 2338 & 32.8 \\
\hline NGC 0520 & $012435.6+034733$ & Pec & 99.0 & $\mathrm{SF} / \mathrm{AGN}$ & 0.04 & 11.98 & 1.35 & -20.88 & 2278 & 37.7 \\
\hline NGC 0584 & $013120.7-065205$ & $\mathrm{E} 4$ & -5.0 & $\ldots$ & 0.04 & 10.84 & 1.68 & -20.67 & 1802 & 20.1 \\
\hline NGC 0628 & $013641.8+154701$ & SAc & 5.0 & $\cdots$ & 0.10 & 10.92 & 1.16 & -18.39 & 657 & 7.3 \\
\hline NGC 0660 & $014302.4+133843$ & $\mathrm{SB}(\mathrm{s}) \mathrm{a}$ & 1.0 & $\mathrm{SF} / \mathrm{AGN}$ & 0.07 & 11.98 & 1.50 & -18.59 & 839 & 13.0 \\
\hline III Zw 035 & $014430.5+170609$ & Pec & $\cdots$ & $\mathrm{SF} / \mathrm{AGN}$ & 0.08 & 15.17 & 1.34 & -20.12 & 8214 & 114.0 \\
\hline NGC 0695 & $015114.4+223456$ & So & -2.0 & SF & 0.10 & 13.50 & 1.03 & -22.16 & 9743 & 135.4 \\
\hline NGC 0750 & $015732.7+331227$ & Pec & -5.0 & $\cdots$ & 0.08 & 12.80 & 1.76 & -21.49 & 5156 & 72.1 \\
\hline NGC 0855 & $021403.5+275238$ & $\mathrm{E}$ & -5.0 & SF & 0.09 & 12.52 & 1.06 & -17.41 & 592 & 9.7 \\
\hline NGC 1068 & $024240.7-000047$ & (R)SA(rs)b & 3.0 & AGN & 0.05 & 9.41 & 1.17 & -21.67 & 1139 & 16.5 \\
\hline Arp 118 & $025510.8-001048$ & Pec & -4.3 & $\mathrm{SF} / \mathrm{AGN}$ & 0.07 & 12.79 & 1.44 & -22.63 & 8634 & 119.9 \\
\hline NGC 1144 & $025512.2-001101$ & Pec & -4.3 & $\mathrm{SF}$ & 0.07 & 13.30 & 1.30 & -22.11 & 8634 & 119.9 \\
\hline NGC 1275 & $031948.1+413042$ & $\mathrm{Pec}$ & 99.0 & $\cdots$ & 0.20 & 11.21 & 1.15 & -23.14 & 5276 & 74.5 \\
\hline NGC 1614 & $043400.0-083445$ & $\mathrm{SB}(\mathrm{s}) \mathrm{c}$ & 5.0 & $\mathrm{SF} / \mathrm{AGN}$ & 0.14 & 12.49 & 1.02 & -21.64 & 4766 & 67.4 \\
\hline NGC 2388 & $072853.4+334909$ & Irr & $\cdots$ & SF & 0.08 & 13.62 & 1.72 & -20.34 & 4137 & 62.1 \\
\hline NGC 2403 & $073651.4+653609$ & SABcd & 6.0 & $\cdots$ & 0.09 & 9.29 & 0.89 & -18.18 & 131 & 3.1 \\
\hline NGC 2537 & $081314.4+455931$ & $\mathrm{SB}(\mathrm{s}) \mathrm{m}$ & 9.0 & SF & 0.06 & 11.98 & 0.92 & -17.21 & 419 & 6.9 \\
\hline NGC 2623 & $083824.1+254517$ & Pec & 99.0 & AGN & 0.06 & 13.73 & 1.33 & -20.85 & 5546 & 83.2 \\
\hline IRAS $08572+3915$ & $090025.4+390354$ & Pec & $\ldots$ & $\mathrm{SF} / \mathrm{AGN}$ & 0.03 & 16.37 & 1.16 & -20.63 & 17477 & 249.2 \\
\hline UGC 04881 & $091555.0+441949$ & Pec & $\cdots$ & SF & 0.02 & 14.30 & 1.36 & -21.88 & 11781 & 170.3 \\
\hline NGC 2798 & $091722.8+415959$ & SBa pec & 1.0 & $\mathrm{SF} / \mathrm{AGN}$ & 0.03 & 12.28 & 1.26 & -19.80 & 1726 & 26.2 \\
\hline UGCA 166 & $093402.2+551425$ & Irr & $\cdots$ & SF & 0.06 & 15.76 & 0.17 & -15.20 & 749 & 15.6 \\
\hline UGC 05101 & $093551.5+612112$ & Pec & $\cdots$ & $\mathrm{AGN}$ & 0.05 & 14.85 & 1.54 & -21.33 & 11811 & 170.4 \\
\hline NGC 3049 & $095449.6+091616$ & SBab & 2.0 & SF & 0.06 & 11.91 & 0.85 & -19.97 & 1468 & 23.9 \\
\hline NGC 3079 & $100157.8+554048$ & $\mathrm{SB}(\mathrm{s}) \mathrm{c}$ & 7.0 & $\mathrm{SF} / \mathrm{AGN}$ & 0.01 & 11.03 & 1.17 & -20.40 & 1109 & 19.4 \\
\hline UGCA 208 & $101628.2+451918$ & Pec & $\cdots$ & AGN & 0.01 & 14.81 & 0.71 & -17.55 & 1648 & 29.8 \\
\hline NGC 3190 & $101805.7+214957$ & SAa pec & 1.0 & $\cdots$ & 0.03 & 11.07 & 1.63 & -21.03 & 1271 & 26.4 \\
\hline NGC 3198 & $101954.9+453258$ & $\mathrm{SBc}$ & 5.0 & SF & 0.01 & 11.13 & 1.21 & -19.54 & 659 & 13.7 \\
\hline NGC 3265 & $103106.8+284748$ & $\mathrm{E}$ & -5.0 & SF & 0.02 & 13.55 & 1.24 & -18.27 & 1319 & 23.2 \\
\hline Mrk 33 & $103231.9+542403$ & Im pec & 10.0 & SF & 0.02 & 12.76 & 0.67 & -19.02 & 1430 & 22.9 \\
\hline NGC 3310 & $103845.8+533012$ & $\mathrm{SAB}(\mathrm{r}) \mathrm{bc}$ & 4.0 & SF & 0.02 & 11.05 & 0.54 & -20.45 & 989 & 20.1 \\
\hline NGC 3351 & $104357.7+114214$ & $\mathrm{SBb}$ & 3.0 & SF & 0.04 & 10.19 & 1.38 & -19.65 & 779 & 9.3 \\
\hline NGC 3379 & 104749.6 +123454 & E0 & -5.0 & $\cdots$ & 0.03 & 10.06 & 1.73 & -20.05 & 911 & 10.6 \\
\hline UGCA 219 & $104905.0+522008$ & $\mathrm{Sc}$ & $\cdots$ & SF & 0.02 & 14.47 & 0.37 & -18.54 & 2398 & 40.5 \\
\hline NGC 3521 & $110548.6-000209$ & SABbc & 4.0 & SF & 0.05 & 9.18 & 1.47 & -20.84 & 809 & 10.1 \\
\hline NGC 3627 & $112015.0+125930$ & $\mathrm{SABb}$ & 3.0 & $\mathrm{SF} / \mathrm{AGN}$ & 0.03 & 10.22 & 1.38 & -19.64 & 727 & 9.4 \\
\hline IC 0691 & $112644.3+590920$ & Irr & $\cdots$ & $\mathrm{SF}$ & 0.01 & 13.90 & 0.78 & -17.97 & 1199 & 23.7 \\
\hline NGC 3690 & $112831.7+583344$ & $\mathrm{Pec}$ & 9.0 & $\mathrm{SF} / \mathrm{AGN}$ & 0.01 & 12.03 & 0.75 & -21.47 & 3117 & 50.6 \\
\hline NGC 3773 & $113813.0+120644$ & SA0 & -2.0 & SF & 0.03 & 12.80 & 0.69 & -17.57 & 989 & 11.9 \\
\hline Mrk 1450 & $113835.6+575227$ & Irr & $\cdots$ & SF & 0.01 & 15.34 & 0.70 & -16.16 & 959 & 20.0 \\
\hline UGC 06665 & $114212.3+002004$ & $\mathrm{Sb}$ & 3.0 & SF & 0.04 & 13.85 & 0.60 & -20.83 & 5576 & 87.4 \\
\hline NGC 3870 & $114556.6+501157$ & So & -2.0 & SF & 0.01 & 13.10 & 0.82 & -18.37 & 749 & 19.8 \\
\hline UM 461 & $115133.1-022222$ & Irr & $\cdots$ & SF & 0.03 & 15.35 & 0.85 & -15.28 & 1049 & 13.4 \\
\hline UGC 06850 & $115237.4-022809$ & Irr & 99.0 & SF & 0.04 & 14.14 & 0.46 & -16.48 & 1049 & 13.4 \\
\hline NGC 3938 & $115249.4+440715$ & SAc & 5.0 & $\cdots$ & 0.01 & 10.97 & 1.17 & -19.66 & 809 & 13.4 \\
\hline NGC 4088 & $120534.2+503221$ & $\mathrm{SAB}(\mathrm{rs}) \mathrm{bc}$ & 4.0 & SF & 0.02 & 10.79 & 1.14 & -20.69 & 749 & 19.8 \\
\hline NGC 4125 & $120805.9+651028$ & E6 pec & -5.0 & $\cdots$ & 0.03 & 10.79 & 1.75 & -21.09 & 1356 & 23.9 \\
\hline NGC 4138 & $120929.8+434107$ & $\mathrm{SA}(\mathrm{r}) 0$ & -1.0 & $\mathrm{SF} / \mathrm{AGN}$ & 0.01 & 11.54 & 1.44 & -19.16 & 899 & 13.8 \\
\hline NGC 4168 & $121217.3+131219$ & $\mathrm{E}$ & -5.0 & $\cdots$ & 0.03 & 11.45 & 1.67 & -20.99 & 2215 & 30.9 \\
\hline NGC 4194 & $121409.6+543136$ & $\mathrm{Pec}$ & 10.0 & $\mathrm{SF} / \mathrm{AGN}$ & 0.02 & 12.82 & 0.97 & -20.26 & 2428 & 41.5 \\
\hline Haro 06 & $121518.4+054539$ & Irr & -5.0 & SF & 0.03 & 14.77 & 0.55 & -15.89 & 2008 & 13.7 \\
\hline NGC 4254 & $121849.6+142460$ & SAc & 5.0 & SF & 0.04 & 10.49 & 1.07 & -20.58 & 2398 & 16.5 \\
\hline NGC 4321 & $122254.8+154919$ & SABbc & 4.0 & SF & 0.03 & 10.32 & 1.15 & -20.45 & 1558 & 14.3 \\
\hline NGC 4365 & $122428.3+071904$ & $\mathrm{E}$ & -5.0 & $\cdots$ & 0.03 & 10.40 & 1.75 & -21.41 & 1243 & 23.1 \\
\hline NGC 4387 & $122541.7+124838$ & $\mathrm{E}$ & -5.0 & $\cdots$ & 0.02 & 12.62 & 1.65 & -18.65 & 472 & 18.0 \\
\hline NGC 4385 & $122542.8+003422$ & $\mathrm{SB}(\mathrm{rs}) 0$ & -1.0 & $\mathrm{SF} / \mathrm{AGN}$ & 0.03 & 11.94 & 1.04 & -20.74 & 2128 & 34.7 \\
\hline NGC 4450 & $122829.6+170506$ & SAab & 2.0 & AGN & 0.05 & 10.39 & 1.61 & -20.68 & 1954 & 16.5 \\
\hline NGC 4458 & $122857.6+131431$ & $\mathrm{E}$ & -5.0 & $\cdots$ & 0.02 & 12.59 & 1.56 & -18.46 & 1307 & 16.3 \\
\hline NGC 4473 & $122948.9+132546$ & $\mathrm{E}$ & -5.0 & $\cdots$ & 0.03 & 10.69 & 1.68 & -20.22 & 2244 & 15.3 \\
\hline NGC 4486 & $123049.4+122328$ & $\mathrm{E}$ & -4.0 & $\cdots$ & 0.02 & 9.44 & 1.75 & -21.67 & 635 & 16.7 \\
\hline NGC 4536 & $123427.0+021117$ & $\mathrm{SABbc}$ & 4.0 & SF & 0.02 & 11.17 & 1.22 & -19.62 & 1808 & 14.4 \\
\hline
\end{tabular}


Table 1

(Continued)

\begin{tabular}{|c|c|c|c|c|c|c|c|c|c|c|}
\hline Name & J2000 Coordinates & Morphology & T-type & $\begin{array}{l}\text { BPT } \\
\text { Class }\end{array}$ & $\begin{array}{c}E(B-V) \\
(\mathrm{mag})\end{array}$ & $\begin{array}{c}g^{\mathrm{a}} \\
(\mathrm{mag})\end{array}$ & $\begin{array}{c}u-g \\
(\mathrm{mag})\end{array}$ & $\begin{array}{c}M_{g}{ }^{\mathrm{a}} \\
(\mathrm{mag})\end{array}$ & $\begin{array}{c}c z \\
\left(\mathrm{~km} \mathrm{~s}^{-1}\right)\end{array}$ & $\begin{array}{c}D_{L} \\
(\mathrm{Mpc})\end{array}$ \\
\hline NGC 4550 & $123530.6+121315$ & $\mathrm{E}$ & -1.5 & $\ldots$ & 0.03 & 12.01 & 1.54 & -19.01 & 381 & 16.0 \\
\hline NGC 4551 & $123538.0+121550$ & $\mathrm{E}$ & -5.0 & $\cdots$ & 0.03 & 12.34 & 1.70 & -18.70 & 1172 & 16.2 \\
\hline NGC 4552 & $123539.8+123323$ & $\mathrm{E}$ & -5.0 & $\cdots$ & 0.04 & 10.81 & 1.81 & -20.20 & 340 & 15.9 \\
\hline NGC 4559 & $123557.6+275736$ & SABcd & 6.0 & SF & 0.02 & 10.92 & 0.97 & -19.14 & 807 & 10.3 \\
\hline NGC 4569 & $123649.8+130947$ & SABab & 2.0 & $\mathrm{AGN}$ & 0.04 & 9.72 & 1.43 & -21.37 & -235 & 16.5 \\
\hline NGC 4579 & $123743.5+114905$ & $\mathrm{SABb}$ & 3.0 & $\mathrm{AGN}$ & 0.03 & 10.46 & 1.60 & -20.62 & 1517 & 16.5 \\
\hline NGC 4594 & $123959.4-113723$ & SAa & 1.0 & $\cdots$ & 0.06 & 9.38 & 1.80 & -20.46 & 1024 & 9.3 \\
\hline NGC 4625 & $124152.7+411626$ & SABm pec & 9.0 & SF & 0.01 & 12.52 & 1.03 & -17.29 & 599 & 9.2 \\
\hline NGC 4621 & $124202.2+113849$ & $\mathrm{E}$ & -5.0 & $\cdots$ & 0.04 & 10.09 & 1.61 & -20.77 & 410 & 14.9 \\
\hline NGC 4631 & $124207.8+323234$ & SBd & 7.0 & AGN & 0.03 & 9.99 & 0.91 & -19.41 & 606 & 7.6 \\
\hline NGC 4660 & $124432.0+111126$ & $\mathrm{E}$ & -5.0 & $\cdots$ & 0.03 & 11.59 & 1.61 & -19.29 & 1083 & 15.0 \\
\hline NGC 4670 & $124517.1+270731$ & $\mathrm{SB}(\mathrm{s}) 0 / \mathrm{a}$ & $\cdots$ & SF & 0.01 & 12.45 & 0.53 & -19.37 & 1079 & 23.2 \\
\hline NGC $4676 \mathrm{~A}$ & $124609.2+304355$ & Pec & -2.0 & $\mathrm{AGN}$ & 0.01 & 13.96 & 1.34 & -21.08 & 6625 & 101.9 \\
\hline NGC 4725 & $125026.6+253003$ & SABab pec & 2.0 & $\ldots$ & 0.02 & 10.80 & 1.65 & -19.58 & 1206 & 11.9 \\
\hline NGC 4826 & $125643.6+214059$ & SAab & 2.0 & $\mathrm{SF} / \mathrm{AGN}$ & 0.05 & 8.93 & 1.47 & -20.44 & 408 & 7.5 \\
\hline NGC 4860 & $125903.9+280725$ & $\mathrm{E}$ & -5.0 & $\ldots$ & 0.02 & 13.88 & 1.84 & -21.03 & 7980 & 95.1 \\
\hline NGC 4889 & $130008.1+275837$ & E4 & -4.0 & $\cdots$ & 0.01 & 12.12 & 1.77 & -22.75 & 6495 & 93.8 \\
\hline IC 4051 & $130051.5+280234$ & $\mathrm{E}$ & -5.0 & $\ldots$ & 0.01 & 14.03 & 1.84 & -21.43 & 8793 & 122.0 \\
\hline NGC 4926 & $130153.7+273728$ & $\mathrm{E}$ & -3.0 & $\cdots$ & 0.01 & 13.08 & 1.79 & -22.18 & 7887 & 111.2 \\
\hline NGC 5033 & $131327.5+363538$ & SAc & 5.0 & $\mathrm{SF} / \mathrm{AGN}$ & 0.02 & 11.15 & 1.40 & -19.70 & 875 & 14.8 \\
\hline IC 0860 & $131503.5+243708$ & $\mathrm{Sa}$ & $\cdots$ & AGN & 0.01 & 14.00 & 1.57 & -19.71 & 3357 & 55.6 \\
\hline UGC $08335 \mathrm{NW}$ & $131530.7+620745$ & Pec & $\cdots$ & $\mathrm{SF} / \mathrm{AGN}$ & 0.02 & 14.82 & 1.15 & -20.84 & 9233 & 136.1 \\
\hline UGC 08335 SE & $131535.1+620729$ & Pec & $\cdots$ & $\mathrm{SF} / \mathrm{AGN}$ & 0.02 & 14.95 & 1.02 & -20.73 & 9323 & 137.3 \\
\hline NGC 5055 & $131549.3+420146$ & SAbc & 4.0 & $\ldots$ & 0.03 & 9.71 & 1.31 & -19.75 & 484 & 7.8 \\
\hline IC 0883 & $132035.3+340822$ & Pec & 10.0 & $\mathrm{SF} / \mathrm{AGN}$ & 0.01 & 14.34 & 1.26 & -20.79 & 6985 & 106.9 \\
\hline NGC 5104 & $132123.2+002033$ & $\mathrm{Sa}$ & 1.0 & $\mathrm{SF} / \mathrm{AGN}$ & 0.03 & 13.81 & 1.49 & -20.92 & 5576 & 88.3 \\
\hline NGC 5194 & $132952.7+471143$ & SABbc pec & 4.0 & $\mathrm{SF} / \mathrm{AGN}$ & 0.05 & 9.88 & 1.13 & -19.53 & 449 & 7.6 \\
\hline NGC 5195 & $132959.6+471558$ & SB0 pec & 90.0 & $\mathrm{SF} / \mathrm{AGN}$ & 0.05 & 10.74 & 1.53 & -18.67 & 465 & 7.6 \\
\hline NGC 5256 & $133817.4+481638$ & Pec & 99.0 & AGN & 0.02 & 13.48 & 1.20 & -22.01 & 8364 & 125.2 \\
\hline NGC 5257 & $133952.9+005024$ & $\mathrm{SAB}(\mathrm{s}) \mathrm{b}$ & 3.0 & SF & 0.03 & 13.11 & 0.88 & -22.00 & 6805 & 105.8 \\
\hline NGC 5258 & $133957.7+004951$ & $\mathrm{SA}(\mathrm{s}) \mathrm{b}$ & 3.0 & SF & 0.03 & 13.09 & 1.18 & -22.01 & 6745 & 104.9 \\
\hline UGC 08696 & $134442.1+555313$ & Pec & 99.0 & AGN & 0.01 & 14.67 & 1.16 & -21.44 & 11332 & 165.9 \\
\hline Mrk 1490 & $141943.3+491412$ & $\mathrm{Sa}$ & $\cdots$ & $\mathrm{SF} / \mathrm{AGN}$ & 0.02 & 14.99 & 1.50 & -20.34 & 7674 & 115.5 \\
\hline NGC 5653 & $143010.4+311256$ & $\left(\mathrm{R}^{\prime}\right) \mathrm{SA}(\mathrm{rs}) \mathrm{b}$ & 3.0 & SF & 0.02 & 12.52 & 1.09 & -21.30 & 3567 & 58.7 \\
\hline Mrk 0475 & $143905.5+364821$ & Irr & $\cdots$ & SF & 0.01 & 15.49 & 0.79 & -14.75 & 569 & 11.2 \\
\hline NGC 5713 & $144011.4-001720$ & SABbc pec & 4.0 & SF & 0.04 & 11.30 & 1.06 & -21.03 & 1899 & 29.4 \\
\hline UGC $09618 \mathrm{~S}$ & $145700.3+243624$ & $\mathrm{Sc}$ & $\cdots$ & $\mathrm{SF} / \mathrm{AGN}$ & 0.05 & 14.49 & 1.12 & -21.38 & 10043 & 150.2 \\
\hline UGC 09618 & $145700.3+243647$ & Pec & $\cdots$ & $\mathrm{SF} / \mathrm{AGN}$ & 0.05 & 13.85 & 1.24 & -22.06 & 10103 & 151.0 \\
\hline UGC $09618 \mathrm{~N}$ & $145700.7+243703$ & $\mathrm{Sb}$ & $\cdots$ & SF & 0.05 & 14.68 & 1.48 & -21.25 & 10103 & 151.0 \\
\hline NGC 5866 & $150629.5+554546$ & So & -1.0 & $\cdots$ & 0.02 & 10.64 & 1.63 & -20.29 & 672 & 15.4 \\
\hline CGCG 049-057 & $151313.1+071332$ & Irr & $\cdots$ & $\mathrm{SF} / \mathrm{AGN}$ & 0.04 & 15.02 & 1.65 & -18.98 & 3897 & 63.3 \\
\hline NGC 5953 & $153432.4+151138$ & $\mathrm{Sa}$ & 1.0 & $\mathrm{SF} / \mathrm{AGN}$ & 0.04 & 12.49 & 1.25 & -20.19 & 1978 & 34.6 \\
\hline IC 4553 & $153457.2+233011$ & Pec & $\cdots$ & AGN & 0.05 & 13.59 & 1.58 & -21.05 & 5426 & 85.2 \\
\hline UGCA 410 & $153704.2+551548$ & Irr & $\cdots$ & SF & 0.02 & 14.95 & 0.67 & -15.99 & 659 & 15.4 \\
\hline NGC 5992 & $154421.5+410511$ & $\mathrm{SBb}$ & $\cdots$ & SF & 0.02 & 13.84 & 0.98 & -21.88 & 9503 & 141.3 \\
\hline NGC 6052 & $160512.8+203232$ & $\mathrm{Pec}$ & 5.0 & SF & 0.08 & 12.96 & 0.64 & -21.38 & 4706 & 74.8 \\
\hline NGC 6090 & $161140.9+522727$ & Pec & $\ldots$ & $\mathrm{SF}$ & 0.02 & 13.89 & 0.74 & -21.67 & 8783 & 130.2 \\
\hline NGC 6240 & $165258.9+022405$ & Pec & 90.0 & $\mathrm{AGN}$ & 0.10 & 13.07 & 1.39 & -22.19 & 7344 & 111.9 \\
\hline IRAS $17208-0014$ & $172322.0-001701$ & $\mathrm{Pec}$ & $\cdots$ & SF & 0.37 & 15.16 & 1.49 & -21.23 & 12831 & 187.9 \\
\hline II Zw 096 & $205724.1+170746$ & Pec & $\cdots$ & SF & 0.10 & 13.49 & 0.63 & -22.42 & 10822 & 154.5 \\
\hline NGC 7331 & $223704.0+342457$ & $\mathrm{SAb}$ & 3.0 & $\mathrm{SF} / \mathrm{AGN}$ & 0.11 & 9.58 & 1.60 & -21.23 & 816 & 14.5 \\
\hline UGC 12150 & $224112.2+341457$ & $\mathrm{SB} 0 / \mathrm{a}$ & $\cdots$ & $\mathrm{SF} / \mathrm{AGN}$ & 0.08 & 14.15 & 1.60 & -20.66 & 6415 & 91.3 \\
\hline CGCG 453-062 & $230456.6+193308$ & $\mathrm{Sab}$ & $\cdots$ & SF & 0.10 & 14.02 & 1.51 & -21.12 & 7524 & 106.0 \\
\hline IC 5298 & $231600.7+253324$ & Pec & $\cdots$ & AGN & 0.09 & 14.11 & 1.39 & -21.21 & 8184 & 115.1 \\
\hline NGC 7585 & $231801.3-043902$ & $(\mathrm{R}) \mathrm{SA}(\mathrm{s}) 0$ & -1.0 & $\cdots$ & 0.08 & 11.39 & 1.65 & -22.09 & 3507 & 50.1 \\
\hline NGC 7591 & $231816.3+063509$ & $\mathrm{SBbc}$ & 4.0 & SF & 0.12 & 13.26 & 1.22 & -20.96 & 4946 & 69.9 \\
\hline NGC 7592 & $231822.1-042458$ & Pec & -0.7 & $\mathrm{SF} / \mathrm{AGN}$ & 0.05 & 13.55 & 0.83 & -21.50 & 7314 & 102.9 \\
\hline NGC 7673 & $232741.2+233521$ & $\left(\mathrm{R}^{\prime}\right) \mathrm{SAc}$ & 5.0 & SF & 0.05 & 12.89 & 0.63 & -20.53 & 3417 & 48.9 \\
\hline NGC 7674 & $232756.7+084644$ & $\mathrm{SA}(\mathrm{r}) \mathrm{bc}$ & 4.0 & $\cdots$ & 0.07 & 12.96 & 1.08 & -22.45 & 8664 & 121.4 \\
\hline NGC 7679 & $232846.6+033041$ & SB0 & -2.0 & $\mathrm{SF} / \mathrm{AGN}$ & 0.08 & 12.53 & 0.91 & -21.74 & 5126 & 72.3 \\
\hline Mrk 0930 & $233158.6+285650$ & Pec & $\cdots$ & SF & 0.15 & 14.57 & 0.46 & -19.82 & 5486 & 77.5 \\
\hline NGC 7714 & $233614.1+020918$ & $\mathrm{SB}(\mathrm{s}) \mathrm{b}$ & 3.0 & $\mathrm{SF} / \mathrm{AGN}$ & 0.08 & 12.44 & 0.64 & -20.54 & 2788 & 39.9 \\
\hline NGC 7771 & $235124.8+200642$ & $\mathrm{SB}(\mathrm{s}) \mathrm{a}$ & 1.0 & SF & 0.09 & 12.25 & 1.39 & -21.65 & 4287 & 60.5 \\
\hline Mrk 0331 & $235126.7+203510$ & $\mathrm{Sa}$ & $\cdots$ & $\mathrm{SF} / \mathrm{AGN}$ & 0.09 & 13.70 & 1.37 & -20.76 & 5546 & 77.9 \\
\hline
\end{tabular}

Note. ${ }^{a}$ For the $g$-band apparent and absolute magnitudes, we approximate total magnitudes by using either the brighter of the NASA-Sloan Atlas Sérsic model fit photometry or our aperture photometry. 
(Skrutskie et al. 2006), and mid-infrared imaging from WISE. When galaxies overlapped the SDSS III or 2MASS image boundaries, we used mosaic images from the NASA-Sloan Atlas (Blanton et al. 2011), the 2MASS Large Galaxy Atlas (Jarrett et al. 2003), or generated new Swarp mosaics (Bertin et al. 2002). For the WISE bands, we used release version 4.1 atlas images from the WISE All Sky Data Release. When available, we utilized Spitzer Infrared Array Camera (IRAC; Fazio et al. 2004), IRS, and MIPS (Rieke et al. 2004) mosaics available from the Spitzer Infrared Nearby Galaxies Survey (SINGS; Kennicutt et al. 2003) or the Spitzer Heritage Archive.

For a small number of galaxies we recombined individual exposures to improve signal to noise and cosmic-ray rejection. In some instances, where multiple images were available for the same galaxy in the same band (e.g., GALEX NUV images from two surveys), we measured photometry using both images to check consistency. The background of the images was estimated using the median of the pixel values that are outside of the aperture. The background in the 2MASS and Spitzer images can vary as a function of position, so we visually inspected all of the images, and for those images with significant background variations we subtracted a model background determined with IRAF's imsurfit task.

We defined our photometric aperture to (where possible) approximate the rectangular apertures used by Kennicutt (1992), Gavazzi et al. (2004), Moustakas \& Kennicutt (2006), and Moustakas et al. (2010) for the optical drift-scan spectrophotometry. In some instances the photometric aperture was reduced in size to mitigate contamination from neighboring sources or improve signal to noise at some wavelengths (particularly the ultraviolet and mid-infrared). The photometric apertures used for each of the galaxies are defined in Table 2. Bright stars are manually flagged, masked, and not included in the aperture photometry presented in this paper. As the masked regions are very small relative to the large apertures used to measure galaxy photometry, we did not attempt to model the galaxy light within the masked regions. For some galaxies the aperture only covers a fraction of the galaxy, and the fraction of light captured in the $g$ band can be estimated by comparing (approximate) total and aperture magnitudes presented in Tables 1 and 3, respectively. However, for more than $70 \%$ of our sample the $g$-band aperture magnitudes are within 0.3 mag or brighter than Sérsic model fit magnitudes provided by the NASA-Sloan Atlas. In this paper we largely use photometry corrected for foreground dust extinction, but in Table 3 the photometry is presented without dust extinction corrections (as the corrections are model dependent). The modeled Milky Way dust extinction for each sample galaxy and band is presented in Table 4 .

Many galaxies in our sample are bright so photon counting noise is likely to be small relative to systematic errors. For the brightest galaxies in our sample, we assumed the uncertainties had a floor of $0.05 \mathrm{mag}$ in the SDSS and 2MASS bands, and we assumed the uncertainties in the other bands had a floor of $0.10 \mathrm{mag}$. For the fainter galaxies in our sample (i.e., $m_{\mathrm{FUV}}>$ $18, m_{K_{S}}>13$ ), we estimated the uncertainties and corrected for background errors by measuring fluxes at 24 locations (offset from the galaxy position). The distribution of the fluxes in these apertures was used to determine the uncertainties while the median of the fluxes was used to correct for background errors. For a small number of star-forming galaxies, the 2MASS photometry has very large uncertainties so we used the near-infrared photometry of Engelbracht et al. (2008) and Vaduvescu et al. (2005) (when available) for constraining and verifying the spectra.

\subsubsection{Zero-point and Point-spread Function Corrections}

Large aperture photometry will overestimate fluxes if the zero-points have been determined with small apertures that do not capture all the light from relevant standard stars. The Spitzer IRAC imaging was calibrated using 24" diameter aperture photometry (without corrections for flux beyond the aperture) and IRAC large aperture photometry suffers from scattered light within the aperture, which can increase measured galaxy fluxes by $10 \%$ or more (Fazio et al. 2004). To mitigate these issues, we used the IRAC point-spread function (PSF) to determine the fraction of light beyond the aperture and we used the corrections described in the IRAC Instrument Handbook ${ }^{18}$ to model scattered light as a function of aperture area. Swift UVOT photometry was calibrated using $10^{\prime \prime}$ diameter aperture photometry (without corrections for flux beyond the aperture) and we correct for this using approximations to the curves of growth presented in Breeveld et al. (2010). Spitzer IRS peak-up blue and red channel imaging is calibrated using 12 and 13 pixel radius apertures, respectively, and we made small corrections for this using the peak-up imager PSFs. The WISE all sky release zero-points were determined by fitting PSF models to the inner region of the PSF, and consequently there are small zero-point errors for large aperture photometry. To correct for this, we added $0.03,0.04,0.03$, and -0.03 to the measured WISE $W 1$, $W 2, W 3$, and $W 4$ magnitudes, respectively (Jarrett et al. 2012).

For galaxies with small angular sizes, we are effectively measuring total magnitudes and the smallest apertures approach the size of the GALEX, IRS, MIPS, and WISE PSFs. For the ultraviolet and mid-infrared photometry we applied an approximate correction for flux beyond the aperture using radially averaged models of the PSF. For very small apertures the WISE W4 PSF results in PSF corrections of $50 \%$ or more, so for isolated galaxies measured with small spectroscopic extraction apertures (less than $30^{\prime \prime}$ on either side) we increased the $W 4$ aperture size to at least $30^{\prime \prime}$ on a side.

\subsubsection{Photon Coincidence Loss Corrections}

The Swift UVOT is a micro-channel plate intensified CCD and suffers from significant photon coincidence losses (Roming et al. 2005). Coincidence loss corrections have been determined for point sources and a surface brightness limit has been established, below which coincidence losses have a minimal impact on extended source photometry (Breeveld et al. 2010; Hoversten et al. 2011). However, coincidence loss corrections for bright extended sources are not available in the prior literatures. To measure the photon coincidence losses for extended sources, we compared Swift UVOT $U$-band and SDSS $u$-band surface brightness measurements for bright galaxies. Figure 2 compares Swift UVOT and SDSS measurements of the surface brightness for NGC 2403 and NGC 4486, and the impact of photon coincidence losses can be clearly seen. The losses approach $50 \%$ for a surface brightness of $U \sim 20 \mathrm{mag} \operatorname{arcsec}^{-2}$, corresponding to a photon count rate of 0.5 photons s ${ }^{-1} \operatorname{arcsec}^{-2}$. The photon coincidence losses occur at relatively faint magnitudes due to the large $\left(4^{\prime \prime} \times 4^{\prime \prime}\right)$ physical pixel scale of the UVOT detector and the use of five pixels when centroiding photon splashes.

\footnotetext{
18 http://irsa.ipac.caltech.edu/data/SPITZER/docs/irac/ iracinstrumenthandbook/
} 
Table 2

Photometric and Spectroscopic Apertures

\begin{tabular}{|c|c|c|c|c|c|c|c|c|c|}
\hline \multirow[t]{2}{*}{ Name } & \multicolumn{2}{|c|}{ Photometric Aperture } & \multirow{2}{*}{$\begin{array}{c}\text { Optical } \\
\text { Spectrum }\end{array}$} & \multicolumn{2}{|c|}{ Akari Aperture } & \multicolumn{2}{|c|}{ Spitzer IRS SL Aperture } & \multicolumn{2}{|c|}{ Spitzer IRS LL Aperture } \\
\hline & $\begin{array}{c}\text { Size } \\
(\operatorname{arcsec})\end{array}$ & $\begin{array}{l}\text { P.A. } \\
\text { (deg) }\end{array}$ & & $\begin{array}{c}\text { Size } \\
(\operatorname{arcsec})\end{array}$ & $\begin{array}{l}\text { P.A. } \\
\text { (deg) }\end{array}$ & $\begin{array}{c}\text { Size } \\
(\operatorname{arcsec})\end{array}$ & $\begin{array}{l}\text { P.A. } \\
\text { (deg) }\end{array}$ & $\begin{array}{c}\text { Size } \\
(\operatorname{arcsec})\end{array}$ & $\begin{array}{l}\text { P.A. } \\
\text { (deg) }\end{array}$ \\
\hline Arp $256 \mathrm{~S}$ & $40 \times 40$ & 90 & M06 & $29.2 \times 60.0$ & 246 & $25.9 \times 18.5$ & 153 & $61.0 \times 25.4$ & 69 \\
\hline NGC 0337 & $95 \times 55$ & 70 & M10 & $\ldots$ & $\ldots$ & $53.7 \times 31.5$ & 149 & $121.9 \times 71.1$ & 65 \\
\hline CGCG 436-030 & $35 \times 40$ & 90 & M06 & $7.3 \times 60.0$ & 248 & 3.'6 slit & -28 & 10'.6 slit & 67 \\
\hline NGC 0474 & $60 \times 150$ & 90 & M06 & $\ldots$ & $\ldots$ & $\ldots$ & $\ldots$ & $\ldots$ & $\ldots$ \\
\hline NGC 0628 & $346 \times 55$ & 70 & M10 & $\ldots$ & $\ldots$ & $48.1 \times 27.7$ & 152 & $330.2 \times 50.8$ & 68 \\
\hline NGC 0660 & $130 \times 120$ & 90 & M06 & $\ldots$ & $\ldots$ & 3".6 slit & 151 & $10^{\prime \prime} 6$ slit & 248 \\
\hline III Zw 035 & $20 \times 35$ & 90 & M06 & $29.2 \times 60.0$ & 69 & $20.0 \times 35.0$ & -26 & $50.8 \times 40.6$ & -110 \\
\hline NGC 0695 & $60 \times 45$ & 90 & M06 & $25.9 \times 60.0$ & 249 & 3".6 slit & -24 & $10{ }^{\prime} 6$ slit & 71 \\
\hline NGC 0750 & $80 \times 30$ & 90 & M06 & $\ldots$ & $\ldots$ & $\ldots$ & $\ldots$ & $\ldots$ & $\ldots$ \\
\hline NGC 0855 & $86 \times 55$ & 70 & M10 & $\ldots$ & $\ldots$ & $24.0 \times 22.2$ & 156 & $116.9 \times 45.7$ & 73 \\
\hline NGC 1614 & $80 \times 60$ & 90 & M06 & $7.3 \times 60.0$ & 259 & 3".6 slit & -28 & $10^{\prime \prime} 6$ slit & 68 \\
\hline NGC 2388 & $60 \times 30$ & 90 & M06 & $7.3 \times 60.0$ & -82 & $38.9 \times 31.4$ & -174 & $81.3 \times 30.5$ & 102 \\
\hline NGC 2403 & $657 \times 56$ & 127 & M10 & $\ldots$ & $\ldots$ & $129.5 \times 59.2$ & 31 & $549.3 \times 50.8$ & 127 \\
\hline NGC 2537 & $100 \times 60$ & 90 & M06 & $\ldots$ & $\ldots$ & 3."6 slit & 180 & $10^{\prime \prime} 6$ slit & 277 \\
\hline NGC 2623 & $90 \times 40$ & 60 & M06 & $7.3 \times 60.0$ & 104 & $40.7 \times 40.7$ & 11 & $61.0 \times 55.9$ & -73 \\
\hline IRAS $08572+3915$ & $30 \times 20$ & 90 & M06 & $29.2 \times 60.0$ & -72 & 3.'6 slit & 10 & 10'.6 slit & 107 \\
\hline UGC 04881 & $60 \times 40$ & 90 & M06 & $29.2 \times 60.0$ & 110 & 3."6 slit & 197 & 10'.6 slit & 294 \\
\hline NGC 2798 & $84 \times 55$ & 103 & M10 & $\ldots$ & $\ldots$ & $53.7 \times 31.4$ & 23 & $106.7 \times 55.9$ & 120 \\
\hline UGCA 166 & $20 \times 20$ & 90 & M06 & $\ldots$ & $\ldots$ & 3".6 slit & 176 & $10^{\prime \prime} 6$ slit & 272 \\
\hline UGC 05101 & $50 \times 30$ & 90 & M06 & $29.2 \times 60.0$ & 116 & 3."6 slit & 45 & 10'.6 slit & 142 \\
\hline NGC 3049 & $72 \times 55$ & 115 & M10 & $\ldots$ & $\ldots$ & $44.4 \times 24.1$ & 15 & $101.6 \times 50.8$ & 112 \\
\hline NGC 3079 & $100 \times 330$ & 90 & M06 & $\ldots$ & $\ldots$ & $53.6 \times 24.0$ & -160 & $116.8 \times 25.4$ & 116 \\
\hline UGCA 208 & $30 \times 20$ & 90 & M06 & $\ldots$ & $\ldots$ & 3".6 slit & 40 & $10^{\prime \prime} 6$ slit & 137 \\
\hline NGC 3521 & $263 \times 56$ & 110 & M10 & $\ldots$ & $\ldots$ & $57.4 \times 35.2$ & 18 & $254.0 \times 55.9$ & 114 \\
\hline NGC 3627 & $200 \times 55$ & 115 & M10 & $\ldots$ & $\ldots$ & $53.7 \times 29.6$ & 23 & $223.5 \times 55.9$ & 119 \\
\hline IC 0691 & $40 \times 40$ & 90 & M06 & $\ldots$ & $\ldots$ & $25.9 \times 7.4$ & 51 & $71.1 \times 20.3$ & -33 \\
\hline NGC 3690 & $90 \times 60$ & 90 & M06 & $29.2 \times 60.0$ & -52 & $40.7 \times 31.4$ & 57 & $111.8 \times 40.6$ & -27 \\
\hline NGC 3773 & $38 \times 55$ & 115 & M10 & $\ldots$ & $\ldots$ & $24.0 \times 24.0$ & -159 & $35.6 \times 35.6$ & 117 \\
\hline Mrk 1450 & $20 \times 15$ & 90 & M06 & $\ldots$ & $\ldots$ & 3.'6 slit & 197 & $10^{\prime} 6$ slit & 294 \\
\hline UGC 06665 & $30 \times 60$ & 90 & M06 & $\ldots$ & $\ldots$ & 3." 6 slit & 197 & $10^{\prime \prime} 6$ slit & 294 \\
\hline NGC 3870 & $50 \times 40$ & 90 & M06 & $\ldots$ & $\ldots$ & $29.6 \times 7.4$ & 51 & $61.0 \times 20.3$ & -33 \\
\hline UM 461 & $25 \times 20$ & 90 & M06 & $\ldots$ & $\ldots$ & 3.'6 slit & 197 & 10'.6 slit & 294 \\
\hline UGC 06850 & $36 \times 40$ & 90 & M06 & $\ldots$ & $\ldots$ & 3."6 slit & 197 & $10^{\prime \prime} 6$ slit & 294 \\
\hline NGC 3938 & $177 \times 56$ & 133 & M10 & $\ldots$ & $\ldots$ & $49.9 \times 29.6$ & 37 & $49.9 \times 29.6$ & 37 \\
\hline NGC 4088 & $140 \times 300$ & 135 & M06 & $\ldots$ & $\ldots$ & $48.1 \times 49.9$ & -149 & $127.0 \times 66.0$ & 128 \\
\hline NGC 4125 & $190 \times 56$ & 90 & M10 & $\ldots$ & $\ldots$ & $24.0 \times 24.1$ & 173 & $24.0 \times 24.1$ & 173 \\
\hline NGC 4138 & $60 \times 120$ & 90 & M06 & $\ldots$ & $\ldots$ & $50.0 \times 51.8$ & -151 & $96.5 \times 55.9$ & 125 \\
\hline NGC 4168 & $105 \times 84$ & 122 & G04 & $\ldots$ & $\ldots$ & .. & $\ldots$ & $\ldots$ & $\ldots$ \\
\hline NGC 4194 & $125 \times 30$ & 165 & M06 & $34.8 \times 60.0$ & -53 & 3.' 6 slit & 178 & $10{ }^{\prime} 6$ slit & 274 \\
\hline Haro 06 & $30 \times 20$ & 90 & M06 & $\ldots$ & $\ldots$ & $9.2 \times 7.4$ & 22 & $35.6 \times 20.3$ & -62 \\
\hline NGC 4254 & $177 \times 55$ & 120 & M10 & $\ldots$ & $\ldots$ & $49.9 \times 35.2$ & 19 & $208.3 \times 50.8$ & 115 \\
\hline NGC 4321 & $245 \times 56$ & 121 & M10 & $\ldots$ & $\ldots$ & $53.6 \times 31.5$ & 25 & $254.0 \times 55.9$ & 121 \\
\hline NGC 4365 & $209 \times 148$ & 45 & G04 & $\ldots$ & $\ldots$ & $\ldots$ & $\ldots$ & $\ldots$ & $\ldots$ \\
\hline NGC 4387 & $110 \times 49$ & 140 & G04 & $\ldots$ & $\ldots$ & $\ldots$ & $\ldots$ & $\ldots$ & $\ldots$ \\
\hline NGC 4385 & $100 \times 60$ & 90 & M06 & $\ldots$ & $\ldots$ & 3."6 slit & 196 & $10^{\prime \prime} 6$ slit & 293 \\
\hline NGC 4450 & $173 \times 55$ & 120 & M10 & $\ldots$ & $\ldots$ & $48.1 \times 29.6$ & 31 & $48.1 \times 29.6$ & 31 \\
\hline NGC 4458 & $108 \times 91$ & 11 & G04 & $\ldots$ & $\ldots$ & $\ldots$ & $\ldots$ & $\ldots$ & $\ldots$ \\
\hline NGC 4473 & $242 \times 120$ & 90 & G04 & $\ldots$ & $\ldots$ & $\ldots$ & $\ldots$ & $\ldots$ & $\ldots$ \\
\hline
\end{tabular}


Table 2

(Continued)

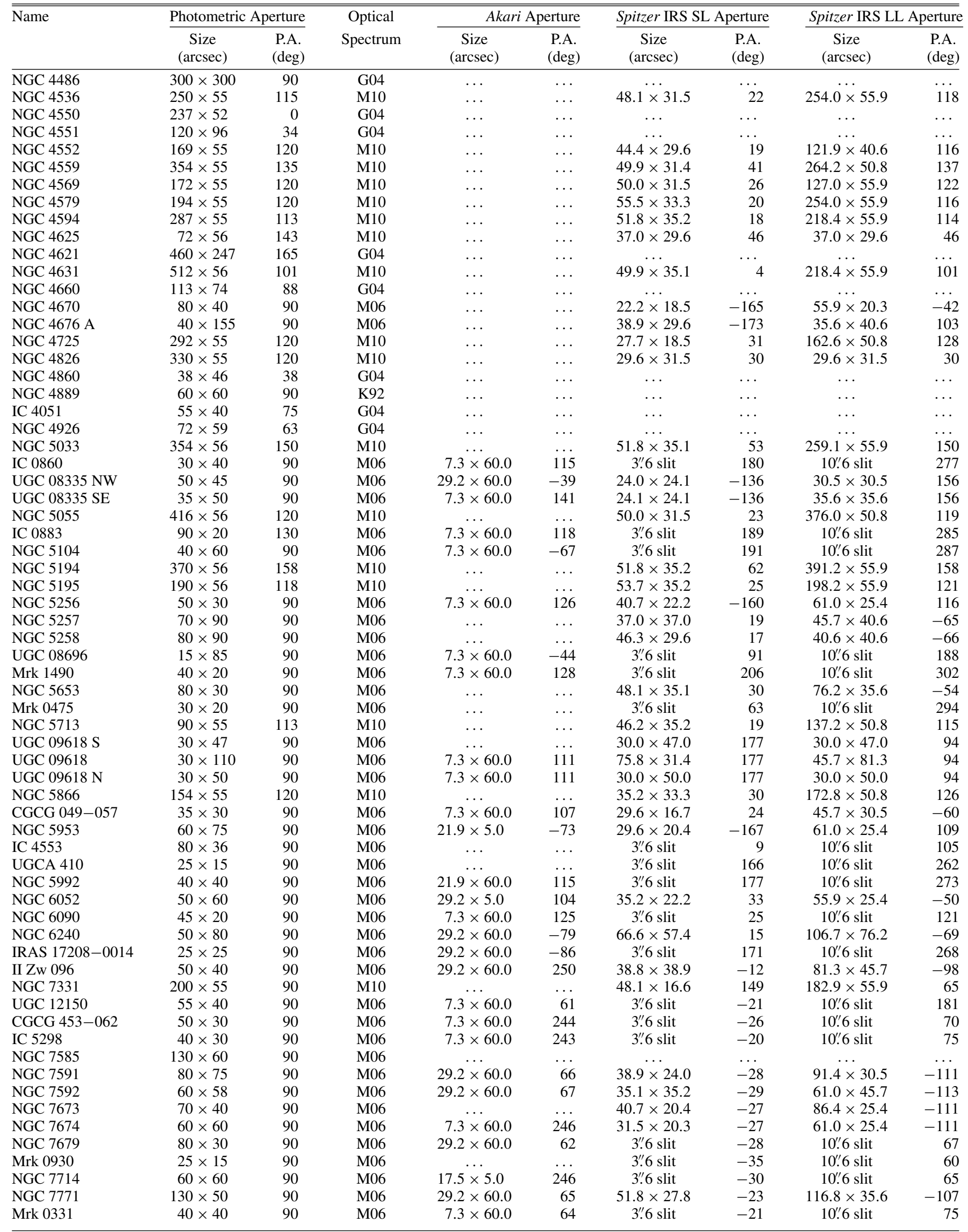


Table 3

AB Magnitudes for the Sample with Neither Foreground Nor Intrinsic Dust Extinction Corrections

\begin{tabular}{|c|c|c|c|c|c|c|c|c|c|}
\hline Name & $\begin{array}{c}\text { FUV } \\
r \\
W 2\end{array}$ & $\begin{array}{c}U V W 2 \\
i \\
{[5.8]}\end{array}$ & $\begin{array}{c}\text { UVM2 } \\
z \\
{[8.0]}\end{array}$ & $\begin{array}{c}\text { NUV } \\
J \\
W 3\end{array}$ & $\begin{array}{c}U V W 1 \\
H \\
P U I \text { Blue }\end{array}$ & $\begin{array}{c}U \\
K_{S} \\
W 4\end{array}$ & $\begin{array}{c}u \\
W 1 \\
W 4^{\prime}\end{array}$ & $\begin{array}{c}g \\
{[3.6]} \\
P U I \text { Red }\end{array}$ & $\begin{array}{c}V \\
{[4.5]} \\
{[24]}\end{array}$ \\
\hline \multirow[t]{3}{*}{ Arp $256 \mathrm{~N}$} & $16.26 \pm 0.10$ & $\cdots$ & $\cdots$ & $15.91 \pm 0.10$ & $\ldots$ & $\ldots$ & $15.31 \pm 0.05$ & $14.43 \pm 0.05$ & $\cdots$ \\
\hline & $14.03 \pm 0.05$ & $13.77 \pm 0.05$ & $13.62 \pm 0.05$ & $13.33 \pm 0.05$ & $13.28 \pm 0.05$ & $13.34 \pm 0.05$ & $13.83 \pm 0.10$ & $13.85 \pm 0.10$ & $14.22 \pm 0.10$ \\
\hline & $14.34 \pm 0.10$ & $13.12 \pm 0.10$ & $11.98 \pm 0.10$ & $12.06 \pm 0.10$ & $\ldots$ & $\ldots$ & $\ldots$ & $\ldots$ & $11.23 \pm 0.10$ \\
\hline \multirow[t]{3}{*}{ Arp $256 \mathrm{~S}$} & $16.37 \pm 0.10$ & $\cdots$ & $\ldots$ & $16.00 \pm 0.10$ & $\ldots$ & $\cdots$ & $15.26 \pm 0.05$ & $14.48 \pm 0.05$ & $\cdots$ \\
\hline & $14.05 \pm 0.05$ & $13.80 \pm 0.05$ & $13.58 \pm 0.05$ & $13.25 \pm 0.05$ & $13.03 \pm 0.05$ & $13.15 \pm 0.05$ & $13.28 \pm 0.10$ & $13.16 \pm 0.10$ & $13.40 \pm 0.10$ \\
\hline & $13.49 \pm 0.10$ & $11.92 \pm 0.10$ & $\ldots$ & $10.53 \pm 0.10$ & $\ldots$ & $8.95 \pm 0.10$ & $9.12 \pm 0.10$ & $\ldots$ & $8.90 \pm 0.10$ \\
\hline \multirow[t]{3}{*}{ NGC 0337} & $15.32 \pm 0.10$ & $14.77 \pm 0.10$ & $14.95 \pm 0.10$ & $14.63 \pm 0.10$ & $14.31 \pm 0.10$ & $\ldots$ & $13.37 \pm 0.05$ & $12.36 \pm 0.05$ & $\cdots$ \\
\hline & $11.88 \pm 0.05$ & $11.65 \pm 0.05$ & $11.51 \pm 0.05$ & $11.27 \pm 0.05$ & $11.12 \pm 0.05$ & $11.34 \pm 0.05$ & $11.89 \pm 0.10$ & $11.85 \pm 0.10$ & $12.24 \pm 0.10$ \\
\hline & $12.36 \pm 0.10$ & $11.24 \pm 0.10$ & $10.31 \pm 0.10$ & $10.46 \pm 0.10$ & $10.31 \pm 0.10$ & $9.63 \pm 0.10$ & $9.79 \pm 0.10$ & $\ldots$ & $9.55 \pm 0.10$ \\
\hline \multirow[t]{3}{*}{ CGCG 436-030 } & $17.48 \pm 0.10$ & $\cdots$ & $\ldots$ & $16.98 \pm 0.10$ & $\ldots$ & $\ldots$ & $15.85 \pm 0.05$ & $14.79 \pm 0.05$ & $\cdots$ \\
\hline & $14.34 \pm 0.05$ & $14.03 \pm 0.05$ & $13.87 \pm 0.05$ & $13.46 \pm 0.05$ & $13.15 \pm 0.05$ & $13.17 \pm 0.07$ & $13.39 \pm 0.10$ & $13.21 \pm 0.10$ & $12.94 \pm 0.10$ \\
\hline & $12.96 \pm 0.10$ & $11.74 \pm 0.10$ & $10.62 \pm 0.10$ & $10.54 \pm 0.10$ & $\ldots$ & $8.79 \pm 0.10$ & $8.99 \pm 0.10$ & $\ldots$ & $8.70 \pm 0.10$ \\
\hline \multirow[t]{3}{*}{ NGC 0474} & $19.16 \pm 0.12$ & $\cdots$ & $\cdots$ & $17.14 \pm 0.10$ & $\cdots$ & $\cdots$ & $14.05 \pm 0.05$ & $12.41 \pm 0.05$ & $\cdots$ \\
\hline & $11.66 \pm 0.05$ & $11.25 \pm 0.05$ & $10.99 \pm 0.05$ & $10.67 \pm 0.05$ & $10.59 \pm 0.05$ & $10.74 \pm 0.05$ & $11.44 \pm 0.10$ & $11.52 \pm 0.10$ & $12.02 \pm 0.10$ \\
\hline & $12.12 \pm 0.10$ & $12.30 \pm 0.10$ & $12.91 \pm 0.10$ & $13.32 \pm 0.10$ & $\ldots$ & $14.08 \pm 0.10$ & $14.15 \pm 0.10$ & & $\ldots$ \\
\hline
\end{tabular}

(This table is available in its entirety in a machine-readable form in the online journal. A portion is shown here for guidance regarding its form and content.)

Table 4

Modeled Milky Way Foreground Dust Extinction

\begin{tabular}{|c|c|c|c|c|c|c|c|c|c|}
\hline Name & $\begin{array}{c}A(\mathrm{FUV}) \\
A(r) \\
A(W 2)\end{array}$ & $\begin{array}{c}A(U V W 2) \\
A(i) \\
A([5.8])\end{array}$ & $\begin{array}{c}A(U V M 2) \\
A(z) \\
A([8.0])\end{array}$ & $\begin{array}{c}A(\mathrm{NUV}) \\
A(J) \\
A(W 3)\end{array}$ & $\begin{array}{c}A(U V W 1) \\
A(H) \\
A(P U I \text { Blue })\end{array}$ & $\begin{array}{c}A(U) \\
A\left(K_{S}\right) \\
A(W 4)\end{array}$ & $\begin{array}{c}A(u) \\
A(W 1) \\
A\left(W 4^{\prime}\right)\end{array}$ & $\begin{array}{c}A(g) \\
A([3.6]) \\
A(P U I \text { Red })\end{array}$ & $\begin{array}{c}A(V) \\
A([4.5]) \\
A([24])\end{array}$ \\
\hline \multirow[t]{3}{*}{ Arp $256 \mathrm{~N}$} & 0.27 & 0.27 & 0.29 & 0.27 & 0.20 & 0.16 & 0.15 & 0.12 & 0.10 \\
\hline & 0.08 & 0.06 & 0.05 & 0.025 & 0.017 & 0.012 & 0.006 & 0.006 & 0.005 \\
\hline & 0.005 & 0.004 & 0.003 & 0.002 & 0.001 & 0.001 & 0.001 & 0.001 & 0.001 \\
\hline \multirow[t]{3}{*}{ Arp $256 \mathrm{~S}$} & 0.27 & 0.27 & 0.29 & 0.27 & 0.20 & 0.16 & 0.15 & 0.12 & 0.10 \\
\hline & 0.08 & 0.06 & 0.05 & 0.025 & 0.017 & 0.012 & 0.006 & 0.006 & 0.005 \\
\hline & 0.005 & 0.004 & 0.003 & 0.002 & 0.001 & 0.001 & 0.001 & 0.001 & 0.001 \\
\hline \multirow[t]{3}{*}{ NGC 0337} & 0.84 & 0.87 & 0.90 & 0.86 & 0.65 & 0.49 & 0.48 & 0.37 & 0.30 \\
\hline & 0.25 & 0.19 & 0.15 & 0.080 & 0.054 & 0.037 & 0.021 & 0.019 & 0.015 \\
\hline & 0.015 & 0.012 & 0.009 & 0.006 & 0.004 & 0.003 & 0.003 & 0.003 & 0.003 \\
\hline \multirow[t]{3}{*}{ CGCG 436-030 } & 0.49 & 0.50 & 0.52 & 0.50 & 0.37 & 0.28 & 0.28 & 0.21 & 0.17 \\
\hline & 0.15 & 0.11 & 0.08 & 0.046 & 0.031 & 0.021 & 0.012 & 0.011 & 0.009 \\
\hline & 0.009 & 0.007 & 0.005 & 0.003 & 0.003 & 0.002 & 0.002 & 0.002 & 0.002 \\
\hline \multirow[t]{3}{*}{ NGC 0474} & 0.31 & 0.31 & 0.33 & 0.31 & 0.23 & 0.18 & 0.17 & 0.13 & 0.11 \\
\hline & 0.09 & 0.07 & 0.05 & 0.029 & 0.020 & 0.013 & 0.007 & 0.007 & 0.006 \\
\hline & 0.005 & 0.004 & 0.003 & 0.002 & 0.002 & 0.001 & 0.001 & 0.001 & 0.001 \\
\hline
\end{tabular}

(This table is available in its entirety in a machine-readable form in the online journal. A portion is shown here for guidance regarding its form and content.)

We applied an approximate correction to the photon counts given by

$$
C_{\mathrm{cor}}=\frac{-\ln \left(1-80 \times C_{\mathrm{raw}} \times f t-2\left[80 \times C_{\mathrm{raw}} \times f t\right]^{2}\right)}{80 \times f t(1-d f)},
$$

where $C_{\text {raw }}$ is the counts per second per square arcsecond, $f t$ is the frame time $(0.011088 \mathrm{~s})$ and $d f$ is the deadtime fraction (0.0155844). As this is an approximation, we measured Swift photometry using images with and without the photon coincidence loss corrections applied, and generally only include measurements when the correction to the photometry is less than $0.3 \mathrm{mag}$. (To increase the number of blue compact dwarfs in our sample, we include Swift photometry for Mrk 1450 with corrections up to 0.5 mag.) A consequence of the coincidence loss corrections and the $0.3 \mathrm{mag}$ criterion is much of the Swift optical photometry (including all the $B$ band) is not included in the final sample.

\subsubsection{WISE W4 Filter Curve Correction}

The pre-launch WISE W4 $(22 \mu \mathrm{m})$ filter response curve does not match the on-sky performance of the WISE W4 measurements (Wright et al. 2010). The WISE photometric measurements are calibrated using a network of A stars and $\mathrm{K}-\mathrm{M}$ giants (Jarrett et al. 2011), with typical spectral indices $(\alpha)$ between 1 and 2, where the spectral index is defined by $f_{v} \propto v^{\alpha}$. Hence, by definition, the photometry is well behaved for objects with Rayleigh-Jeans SEDs, including early-type galaxies. Star-forming galaxies and AGNs with SEDs that rise toward longer wavelengths have WISE W4 magnitudes that are at least $10 \%$ brighter than what is expected from Spitzer IRS spectrophotometry and Spitzer $24 \mu \mathrm{m}$ photometry (Wright et al. 2010; Jarrett et al. 2013). As we show in Figure 3, we have been able to measure the anomaly with greater fidelity with our sample, and measure it as a function of $\sim 22 \mu \mathrm{m}$ spectral index. We find the residual is well approximated by

$$
\Delta m_{W 4}=0.035 \times\left(\alpha_{22}-2\right),
$$



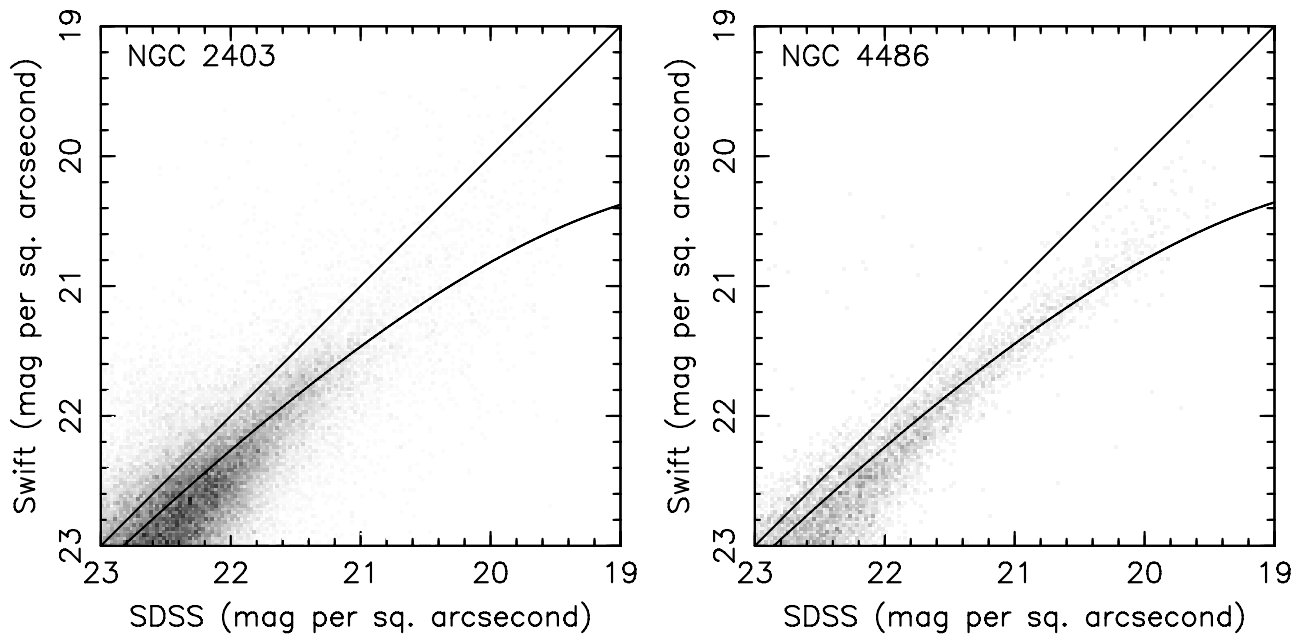

Figure 2. Comparison of Swift $U$-band and SDSS $u$-band photometry for NGC 2403 (left) and NGC 4486 (right) prior to corrections for photon coincidence losses. Surface brightness estimates were determined with single pixels (for the same right ascension and declination) in both the Swift and SDSS images, and $\simeq 0.1$ mag corrections were applied to compensate for the difference between the Swift $U$-band and SDSS $u$-band filters. For extended sources, coincidence losses are significant, even at relatively low surface brightnesses. In both panels the 1:1 line is shown along with the coincidence loss correction curve defined by Equation (3).

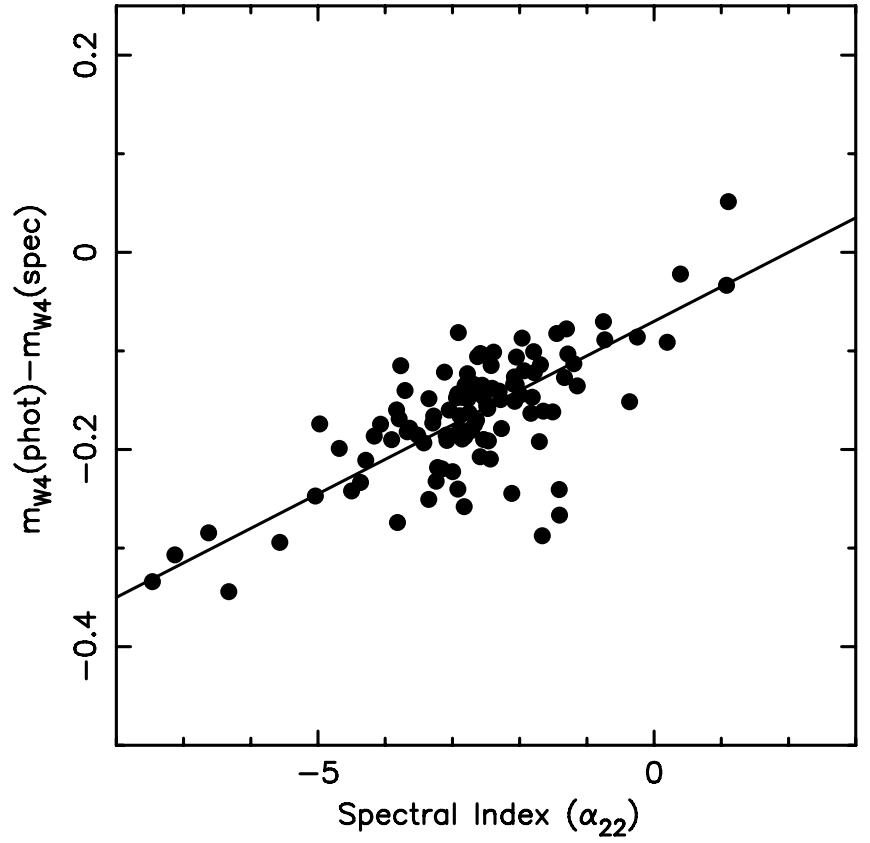

Figure 3. Difference between measured and synthesized $W 4$ magnitudes for galaxies drawn from our sample, plotted as a function of $\sim 22 \mu \mathrm{m}$ spectral index. The pre-launch WISE W4 filter curves does not match the on-sky performance (Wright et al. 2010; Jarrett et al. 2013), so the measured W4 magnitudes are systematically too bright for galaxies with spectra that differ significantly from the Rayleigh-Jeans approximation.

with an rms of 0.05 mag. We present our original and corrected WISE W4 photometry in Table 3, with the latter being denoted by $W 4^{\prime}$.

\subsubsection{The Observed Colors of Nearby Galaxies}

The aperture photometry of the sample galaxies is presented in full in Table 3 and the optical color-magnitude diagram for the sample is plotted in Figure 4. The symbols in Figure 4 are a function of RC3 morphological T-Type (de Vaucouleurs et al. 1991), and sample galaxies that are not in the RC3 have almost exclusively irregular and peculiar morphologies (as shown in Table 1). For comparison, in Figure 4 we also plot the colors and magnitudes of $g<14$ galaxies drawn from the NASA-Sloan Atlas (Blanton et al. 2011). Our sample spans the observed

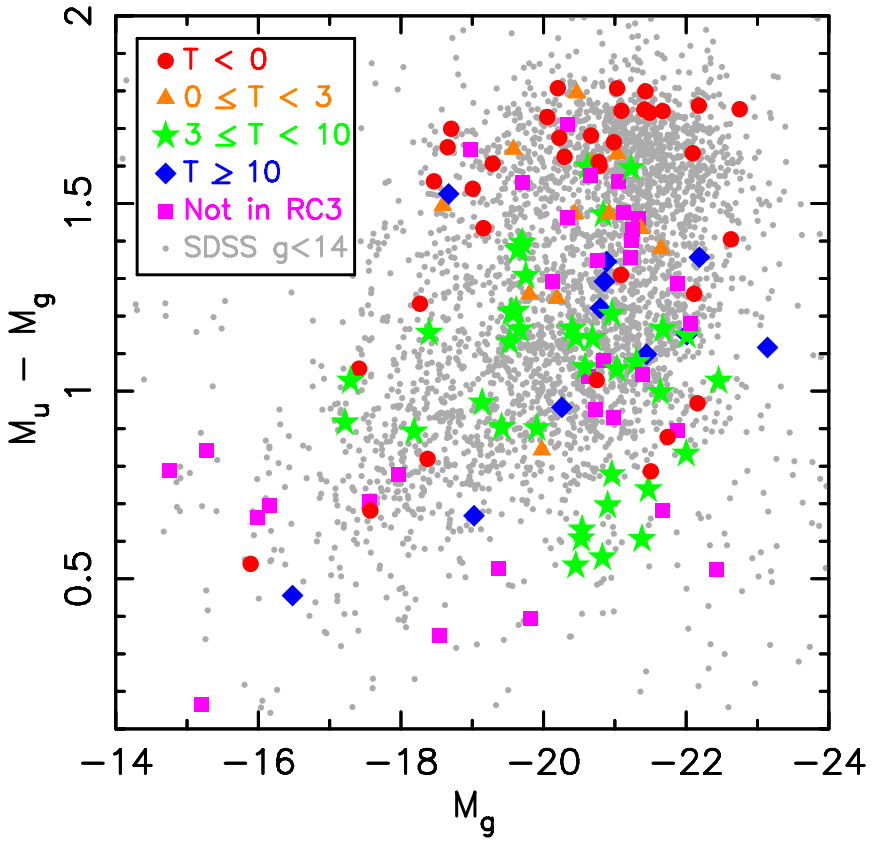

Figure 4. Optical color-magnitude diagram for galaxies in our sample along with $g<14$ galaxies from the NASA-Sloan Atlas. Symbols are a function of RC3 morphological T-Type (de Vaucouleurs et al. 1991), and sample galaxies that are not in the RC3 have almost exclusively irregular and peculiar morphologies. Our sample spans the range of observed colors for $z \sim 0$ galaxies and a broad range of absolute magnitudes.

(A color version of this figure is available in the online journal.)

range of galaxy colors $(0.1<u-g<1.9)$, a broad range of absolute magnitude $\left(-14.7>M_{g}>-23.2\right)$ and a broad range of galaxy types. While our sample spans a broad range of galaxy properties, some galaxy types are not included in the sample (e.g., ultra-compact dwarf galaxies) while other galaxy types (such as LIRGs) are over-represented. We caution that while galaxy morphological types are correlated with galaxy spectra, there are outliers including elliptical galaxies with star formation (e.g., NGC 855) and disk galaxies with low star formation rates (e.g., NGC 4450).

In Figure 5 we plot the optical colors of sample galaxies and NASA-Sloan Atlas galaxies. The majority of galaxies fall along 

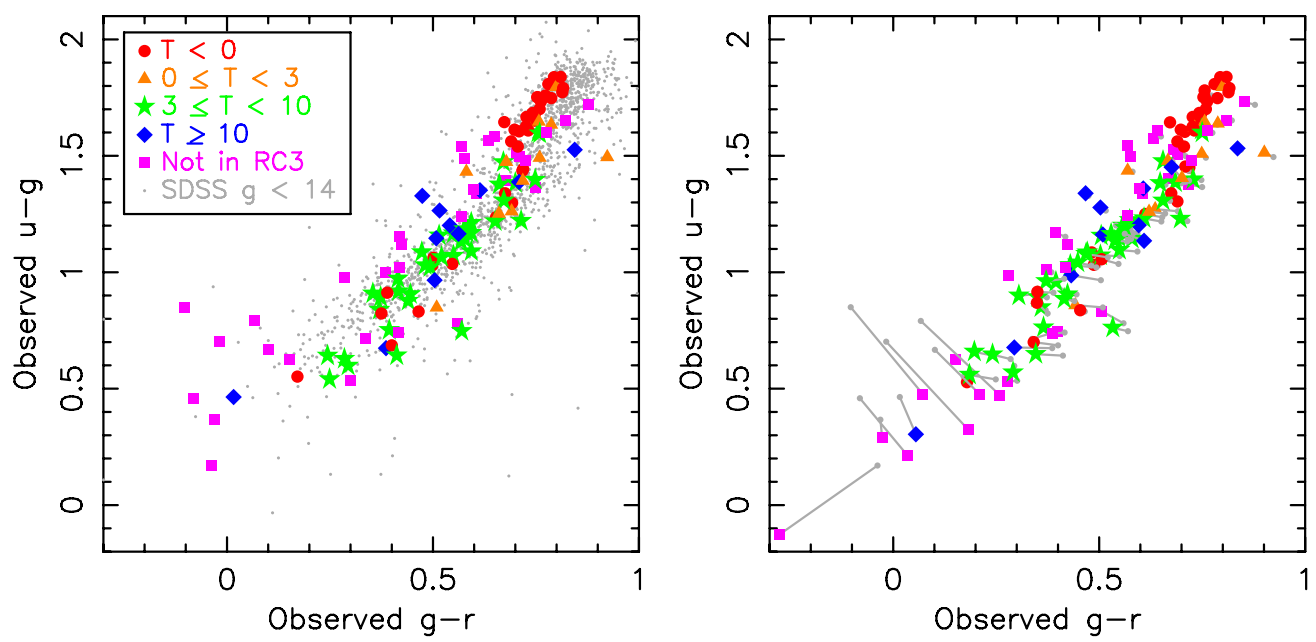

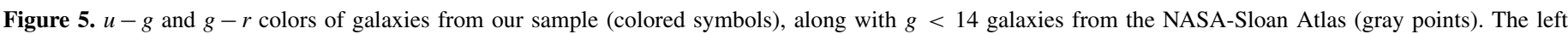

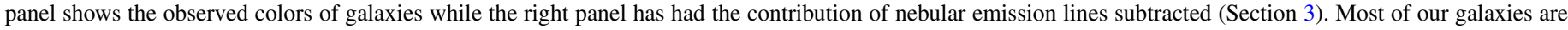

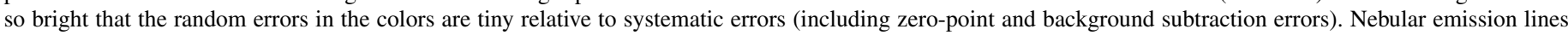

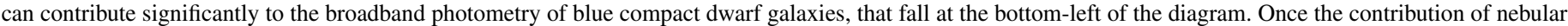

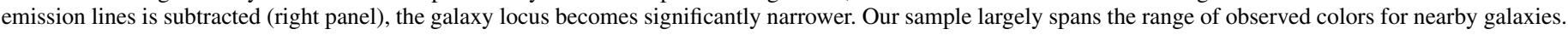
(A color version of this figure is available in the online journal.)

a relatively narrow locus running diagonally across the diagram. Our galaxy locus is slightly offset from the locus of NASASloan Atlas galaxies, due to our use of aperture photometry and the use of Sérsic model fit photometry by NASA-Sloan Atlas. The largest differences between our photometry and the NASA-Sloan Atlas photometry are seen for galaxies where the NASA-Sloan Atlas Sérsic model fits show significant residuals (e.g., NGC 660, NGC 4088) and our aperture does not span the entire galaxy (e.g., NGC 4725, NGC 7331). The optical colors of elliptical galaxies are slightly offset from the locus of starforming galaxies (including dust reddened galaxies), and this offset is known to become more apparent when combinations of optical and near-infrared colors are used (e.g., Cardamone et al. 2010). The bluest galaxies have strong emission lines that contribute significantly to the observed $u g r$ photometry, and these galaxies fan across the lower left of the diagram. Many of the galaxies falling to the right of the galaxy locus are heavily obscured (e.g., NGC 660) while those falling to the left have strong Balmer breaks (e.g., NGC 5992).

The diversity of the sample is illustrated by Figures 6 and 7, where we plot the ultraviolet and infrared colors of sample galaxies. Elliptical galaxies have a broad range of ultraviolet continuum slopes, and this has been known for several decades (e.g., Code \& Welch 1979; Donas et al. 1987, 2007). The distribution of ultraviolet colors tightens as one moves toward the left of Figure 6, with blue dwarf galaxies having a relatively narrow range of ultraviolet colors.

In Figure 7 we plot the infrared colors of the sample galaxies. Galaxies with a Rayleigh-Jeans spectrum at $\sim 4 \mu \mathrm{m}$ fall along the bottom of the figure, with galaxies with weak and strong PAH emission at $\sim 6 \mu \mathrm{m}$ falling on the left and right of the plot respectively. Galaxies that lie above the locus in Figure 7 include AGNs, galaxies with ice absorption at $\simeq 3.1 \mu \mathrm{m}$ and galaxies with hot dust emission. We caution that our large sample does not capture the full diversity of local galaxy populations, as some populations are not included (e.g., ultra-compact dwarfs, low surface brightness galaxies, quasars) and it does not capture the full diversity of other populations (e.g., LIRGs; Howell et al. 2010). However, our galaxy sample does capture much of the diversity of local galaxy populations (as discussed in Section 4)

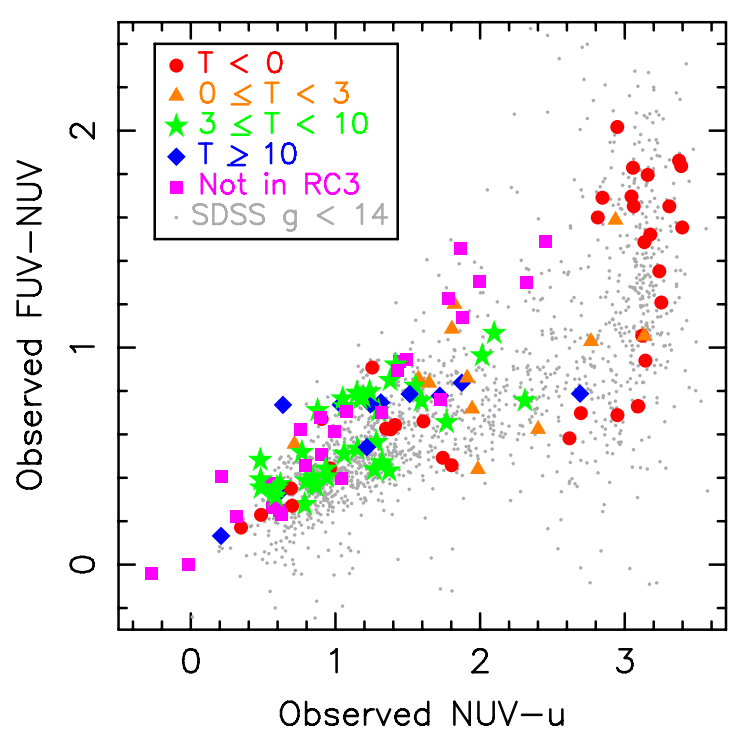

Figure 6. Ultraviolet colors of sample galaxies (colored symbols) and $g<14$ NASA-Sloan Atlas galaxies (gray dots). Early-type galaxies with NUV $-u \sim$ 3.1 have a broad range of far ultraviolet colors. The distribution of galaxy colors tightens as one moves toward low metallicity star-forming galaxies at the bottom-left of the plot.

(A color version of this figure is available in the online journal.)

and illustrates why a relatively small number of galaxy templates can struggle to model the full diversity of local galaxies.

\subsection{Optical Spectra}

The bulk of the optical spectra are from Moustakas \& Kennicutt (2006) and Moustakas et al. (2010), who targeted star-forming galaxies and SINGS galaxies respectively, using both the Bok $2.3 \mathrm{~m}$ telescope on Kitt Peak and the CTIO $1.5 \mathrm{~m}$. These spectra have previously been used by Kennicutt et al. (2009) to calibrate star formation rate indicators and by Moustakas et al. (2010) to measure the gas-phase oxygen abundances of local galaxies. The spectral resolution is $R \sim 650$ and the wavelength coverage spans from 3650 to $6900 \AA$. As the telluric lines near $6870 \AA$ can produce artifacts in 


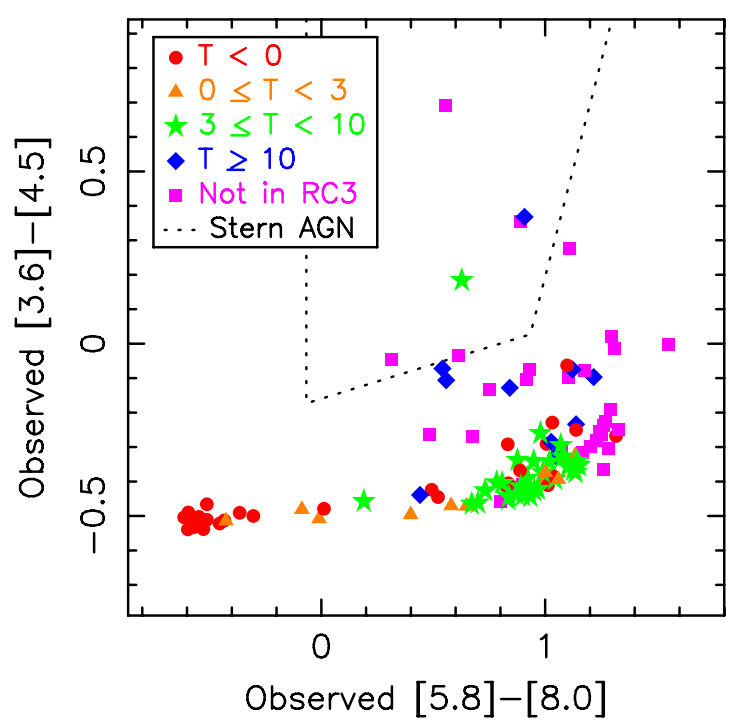

Figure 7. Spitzer IRAC colors for sample galaxies (not all sample galaxies have photometry in all four IRAC bands), along with the Stern et al. (2005) AGN selection criterion (dotted line). Galaxies with SEDs dominated by the Rayleigh-Jeans tail of stellar spectra fall at the bottom-left of the diagram, while galaxies with strong PAH emission fall to the right. Galaxies that are above the locus include AGNs (e.g., NGC 1275), galaxies with ice absorption at $\sim 3 \mu \mathrm{m}$ (e.g., NGC 2623, IC 4553) and galaxies with hot dust emission (e.g., UGCA 166).

(A color version of this figure is available in the online journal.)

the spectra, we truncated the optical spectra at $6830 \AA$ when producing the galaxy SEDs. Observations were obtained by scanning a long-slit perpendicularly back and forth across each galaxy to obtain a luminosity weighted integrated spectrum. In Moustakas et al. (2010) the region used for extracting the integrated spectrum was similar to the region used by Smith et al. (2007b) to obtain 15-38 $\mu \mathrm{m}$ spectra of SINGS galaxies. Relative spectrophotometric accuracy is estimated to be better than $5 \%$ across the optical wavelength range.

Elliptical galaxies have a broad range of ultraviolet continuum slopes (e.g., Code \& Welch 1979; Donas et al. 1987, 2007), and this is not well sampled by the galaxies from Moustakas et al. (2010) that also have SDSS III imaging. To span this region of color-space, we supplemented the sample with driftscan spectrophotometry from Kennicutt (1992) and Gavazzi et al. (2004). Kennicutt (1992) also used the Bok $2.3 \mathrm{~m}$ telescope while Gavazzi et al. (2004) used the $1.93 \mathrm{~m}$ telescope of the Observatoire de Haute Provence, the ESO $3.6 \mathrm{~m}$ telescope, the Loiano $1.52 \mathrm{~m}$ telescope and the San Pedro Martir $2.1 \mathrm{~m}$ telescope. We caution that the spectra from Kennicutt (1992) and Gavazzi et al. (2004) have spectrophotometric accuracy on the order of $\sim 15 \%$, which is poorer than the accuracy of the Moustakas \& Kennicutt (2006) and Moustakas et al. (2010) spectra.

\subsection{Spitzer Infrared Spectra}

Almost all of the galaxies in our sample have low resolution 5-38 $\mu \mathrm{m}$ spectra from the Spitzer IRS. Many of the spectra were obtained for SINGS (Kennicutt et al. 2003; Smith et al. 2007b) and GOALS (Armus et al. 2009; U et al. 2012; Inami et al. 2013; Stierwalt et al. 2013), supplemented by other programs targeting specific galaxy types such as starbursts, low metallicity galaxies, and ellipticals (e.g., Brandl et al. 2006; Wu et al. 2006). For relatively compact galaxies, "stare" observations include a significant fraction of the total flux. When using stare observations we used version five of the Cornell Atlas of Spitzer IRS Sources (Lebouteiller et al. 2011). Tapered extractions were used for most galaxies, but for a small number of compact galaxies PSF weighted extractions were used as these had lower random errors than tapered extractions.

Whenever they were available, we made use of Spitzer IRS spectral maps. For SINGS galaxies spectral maps are available from Smith et al. (2007b) while for the remaining galaxies we used CUBISM (Smith et al. 2007a) to produce new spectral cubes and maps using data downloaded from the Spitzer Heritage Archive. For both the SINGS and new spectral cubes, we extracted spectra using an aperture matched to the optical spectrum or (when this was not possible) we used the largest aperture possible. Extraction apertures and position angles (P.A.s) are summarized in Table 2.

We applied corrections to the IRS short low (SL) and long low (LL) spectra so the spectra were continuous and in good agreement with the photometry. ${ }^{19}$ The IRS low resolution spectra come from four distinct orders; SL2 $(5.25-7.6 \mu \mathrm{m})$, SL1 $(7.5-14 \mu \mathrm{m})$, LL2 $(14.5-20.75 \mu \mathrm{m})$, and LL1 $(20-38.5 \mu \mathrm{m})$. To merge the spectra together, we fitted straight lines to the continua near the overlap regions and then used these fits to determine scalings. To join the SL1 and LL2 spectra, we fitted from $13.65 \mu \mathrm{m}$ to $14.30 \mu \mathrm{m}$ and from $14.0 \mu \mathrm{m}$ to $15.25 \mu \mathrm{m}$, respectively. To join the LL2 and LL1 spectra we fitted from $19.70 \mu \mathrm{m}$ to $20.50 \mu \mathrm{m}$ and from $20.6 \mu \mathrm{m}$ to $22.0 \mu \mathrm{m}$, respective. As poor fits can happen, particularly when dealing with very noisy data, fits were visually inspected and in a small minority of cases manual scalings were applied when merging IRS spectra.

The IRS spectra were scaled to agree with the Spitzer $24 \mu \mathrm{m}$ and WISE $W 4^{\prime}$ photometry. Once this was done, some IRS spectra were significantly offset from the Spitzer $8 \mu \mathrm{m}$ and WISE $W 3$ photometry, in part because the SL1 and SL2 extraction apertures were often smaller than the LL1 and LL2 extraction apertures. We corrected the spectra for this residual, by assuming the residual magnitude difference between the spectra and the photometry varied linearly as a function of wavelength (with the slope being chosen to best match the photometry). This correction is overly simple, but has relatively little impact on the resulting spectra as the (additional) corrections at $8 \mu \mathrm{m}$ are on the order of $30 \%$ for IRS stare observations and often less than $10 \%$ for IRS spectral maps. Galaxies with stare mode spectra were excluded from the sample if the IRS spectrum captured less than $\simeq 25 \%$ of the $8 \mu \mathrm{m}$ flux (some exceptions being made to increase the diversity of the sample). A list of the multiplicative scaling factors used for the Spitzer IRS spectra as a function of wavelength is provided in Table 6 . We caution that for certain science applications, some of our IRS spectra should not be used if the multiplicative scalings are large or vary significantly with wavelength.

\subsection{Akari Infrared Spectra}

Approximately half of the galaxies in the sample have 2-5 $\mu \mathrm{m}$ spectra from the near-infrared channel Akari's IRC (Ohyama et al. 2007; Onaka et al. 2007). These spectra are particularly useful for AGNs and LIRGs, where the $\sim 4 \mu \mathrm{m}$ continuum can include significant non-stellar emission (e.g., NGC 1275, NGC 3690) and the $3.1 \mu \mathrm{m}$ ice feature is sufficiently deep to impact broadband photometry (e.g., NGC 2623, IC 4553). We exclusively used Akari grism spectra, and many 19 As NGC 1068 is saturated in the Spitzer images, we used the corrected
Spitzer photometry of Howell et al. (2007). 


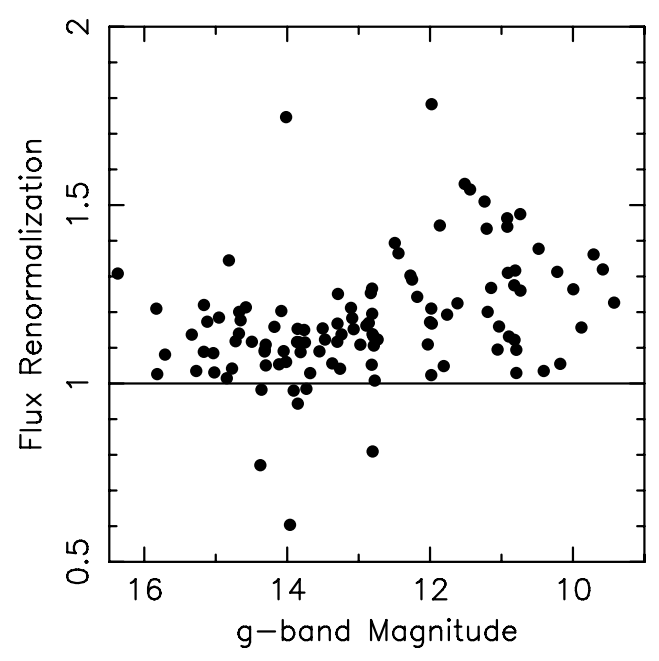

Figure 8. Multiplicative factors used to rescale the optical spectra to match the $g$-band photometry. The optical spectra from Gavazzi et al. (2004) are not absolute flux calibrated so they are not included in this plot. There is a systematic offset of approximately $10 \%$, with larger offsets seen for brighter and more extended galaxies. For large galaxies, the background may be over-subtracted due to the background estimate including some galaxy light. Star formation rate indicators calibrated with these spectra (without renormalization) will underestimate the true star formation rate by $10 \%$ or more.

of the spectra are drawn from Imanishi et al. $(2010)^{20}$ while the remainder were downloaded from JAXA's Data Archives and Transmission System and reduced with the IRC Spectroscopic Toolkit ${ }^{21}$ (Ohyama et al. 2007). IRC frames were darksubtracted, linearity-corrected, flat-field corrected, corrected for positional drift, and combined using the IRC Spectroscopic Toolkit as described in Ohyama et al. (2007). Target galaxies were usually detected automatically within the slit by the toolkit, but in some instances target positions were identified manually. Whenever possible, we used an extraction aperture 20 pixels wide, corresponding to $29^{\prime \prime} .2$, but for some galaxies with low signal-to-noise spectra we had to reduce the width of the extraction aperture. For NGC 7714 the initial Akari reduced spectrum had a systematic error that we removed by remeasuring the background on either side of the extraction aperture. Extraction apertures, slit sizes, and slit P.A.s are detailed in Table 2. For approximately $40 \%$ of the galaxies that had low signal-to-noise grism spectra, we applied a three pixel boxcar smoothing to the data, corresponding to $0.03 \mu \mathrm{m}$.

\section{ASSEMBLING SEDS SPANNING FROM THE ULTRAVIOLET TO THE MID-INFRARED}

Galaxy SEDs were produced by normalizing the observed spectra with our photometry and filling the gaps with spectral coverage with power-law fits or (more frequently) model spectra produced using the Multi-wavelength Analysis of Galaxy Physical Properties code (MAGPHYS; da Cunha et al. 2008). MAGPHYS includes stellar population synthesis models (Bruzual \& Charlot 2003) combined with a self-consistent model of dust emission, dust absorption, and PAH emission.

The multiplicative factors used to renormalize the optical spectra are plotted in Figure 8 and listed in Table 6. Assuming

\footnotetext{
20 The galaxies where we used the Imanishi et al. (2010) Akari spectra are CGCG 049-057, CGCG 436-030, CGCG 453-062, IC 860, IC 883

(UGC 8387), IC 4553 (Arp 220), IC 5298, Mrk 331, Mrk 1490, NGC 1614, NGC 2388, NGC 5104, NGC 6090, NGC 7674, UGC 8335 SE (Mrk 273), UGC 9618 (VV 340), UGC 9618 N, and UGC 12150.

21 http://www.ir.isas.jaxa.jp/ASTRO-F/Observation/DataReduction/IRC/
}

our SDSS $g$-band photometry is valid, the measured optical spectra typically underestimate the fluxes of the true optical spectra by approximately $10 \%$, with larger offsets seen for brighter (and more extended) galaxies. As these underestimates also apply to the nebular emission lines, star formation rate indicators calibrated with the spectra (e.g., Kennicutt et al. 2009) may systematically underestimate the true star formation rates. A possible cause for this systematic underestimation is sky background subtraction using a slit of finite length $(\sim 3.3$ for the Bok $2.3 \mathrm{~m}$ telescope), which may be contaminated by galaxy light for the very largest galaxies in the sample (e.g., NGC 4569). A recalibration of star formation rate indicators (e.g., mid-infrared luminosities between 10 and $24 \mu \mathrm{m}$ ) using our SEDs is beyond the scope of this paper and will be the subject of a future work.

MAGPHYS does not include nebular emission lines, so we produced synthetic optical $u g r$ photometry with the nebular emission lines subtracted, using the emission line fluxes from Moustakas \& Kennicutt (2006) and Moustakas et al. (2010). In cases where [Ne III] $3869 \AA$ was not measured, we used the relation of Pérez-Montero et al. (2007) to approximate the [Ne III] $3869 \AA$ flux using the measured [O III] $5007 \AA$ flux. We show the resulting optical ugr color-colour diagram in the right panel of Figure 5. It is immediately clear from this diagram that the galaxy locus is approximately a straight line once the contribution of emission lines has been removed. Very blue star-forming galaxies that initially have comparable optical colors can move to different regions of the color-color diagram, in part due to differing contributions from [O III] $5007 \AA$ and $\mathrm{H} \alpha$. Consequently, it can be very difficult to determine some properties of emission line galaxies (e.g., stellar masses) when only broadband photometry is available (e.g., Schaerer \& de Barros 2009; Atek et al. 2011).

As we illustrate in Figure 9, the MAGPHYS and observed spectra can approximate each other remarkably well. However, the MAGPHYS and observed spectra do differ from each other, and simply switching from one to the other can produce false spectral breaks. To produce smooth and continuous spectra, we rescaled the MAGPHYS SEDs near the optical, Akari and Spitzer spectra so the models joined the observed spectra smoothly. For example, for each galaxy near $6800 \AA$ we determined a scaling factor by comparing the MAGPHYS and optical spectrum fluxes over a wavelength range corresponding to the last $500 \AA$ of the optical spectrum. We then multiplied the MAGPHYS fluxes by this scaling factor at $\sim 6800 \AA$, and used progressively smaller scaling factors until the MAGPHYS fluxes were unchanged at $10700 \AA$. For galaxies with both Akari and Spitzer spectra, we used linear interpolation to fill the small gap between the Akari and Spitzer spectra. For UGCA 410, MAGPHYS did not approximate the spectra near $6 \mu \mathrm{m}$ so we interpolated between $4.5 \mu \mathrm{m}$ and the Spitzer spectra using a double power law.

For a small number of early-type galaxies we do not have Spitzer IRS spectra and we use power laws to model the spectra of these galaxies at long wavelengths. The $\sim 20 \mu \mathrm{m}$ spectral index is left as a free parameter, as some galaxies do not precisely match a Rayleigh-Jeans spectrum. There are also some galaxies (e.g., AGNs) where the photometry is better matched (over a limited wavelength range) by a power law than a MAGPHYS model (e.g., UGC 5101). For these galaxies we replaced the MAGPHYS model with a power law over the relevant wavelength range. Some galaxies have significant AGN emission in the mid-infrared which cannot be approximated by 

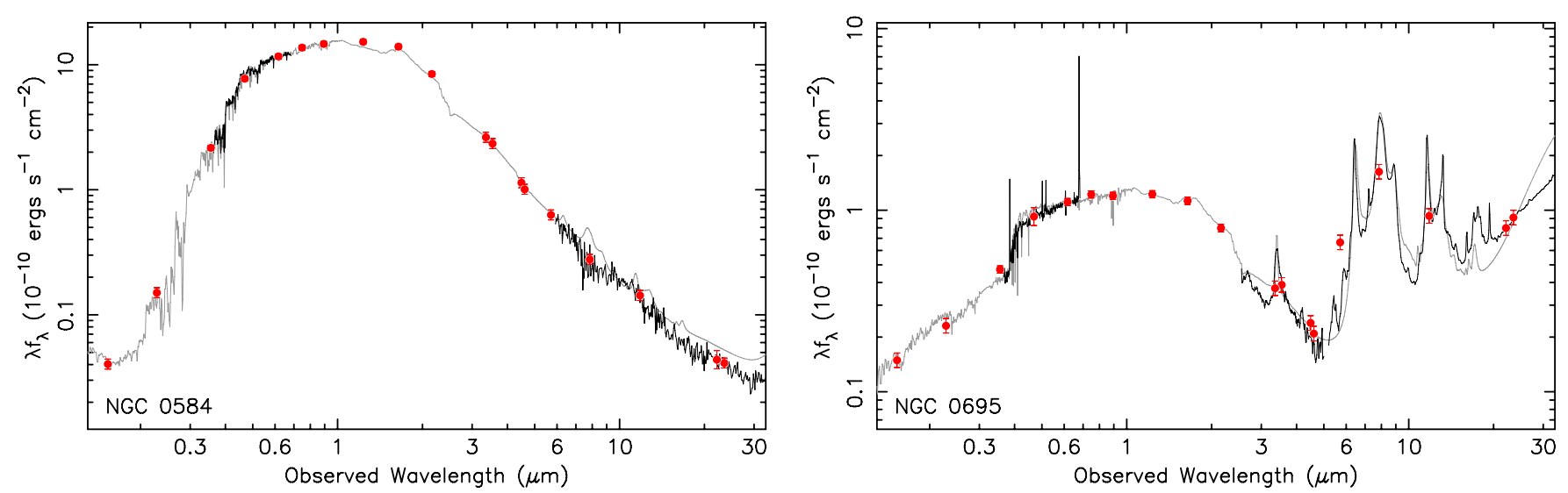

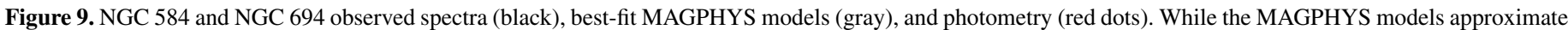

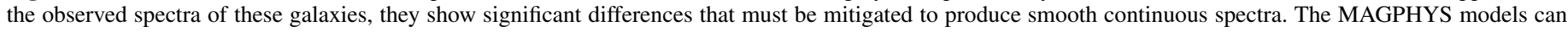
disagree with the observed mid-infrared spectra of galaxies, as our photometry does not accurately constrain the dust temperatures of our galaxies.

(A color version of this figure is available in the online journal.)

MAGPHYS (NGC 1068, NGC 1275, and NGC 6240), so the mid-infrared photometry was excluded when fitting MAGPHYS models to the photometry of these galaxies. Some galaxies have significant AGN emission in the near-infrared that cannot be approximated by MAGPHYS models nor power laws, and (unfortunately) these had to be rejected from the sample (e.g., Mrk 231).

MAGPHYS struggled to fit the ultraviolet photometry of some LIRGs and AGN host galaxies, which is not unexpected given MAGPHYS does not include AGN emission and does not model very complex dust geometries. We applied two corrections to the spectra of CGCG 436-030, IC 860, IC 883, IC 5298, II Zw 96, III Zw 035, IRAS 08572+3915, IRAS 17208-0014, Mrk 331, NGC 2623, NGC 3690, NGC 5256, NGC 6240, NGC 7674, UGC 5101, UGC 8335 S, UGC 8696, and UGC $9168 \mathrm{~N}$ so they were forced to agree with the GALEX FUV and NUV photometry. The two corrections vary linearly with wavelength, with one correction forced to equal zero beyond $2315 \AA$ and the other forced to equal zero beyond $3561 \AA$. The corrections can be as large as $\sim 60 \%$ near the GALEX FUV band, but smaller values are more typical. While the approximations are crude, the resulting spectra should be better than the prior literature (as we discuss in Section 4) and will produce improved broadband colors of LIRGs and AGN host galaxies.

We used the resulting SEDs to determine synthetic photometry, and we compared this with the observed photometry used to normalize and verify the spectra. Any residuals larger than $0.2 \mathrm{mag}$ are flagged and visually inspected. In some cases outliers were removed by improved masking of foreground stars or improved background subtraction. For some galaxies the signal to noise of the optical and Spitzer spectra was low and these were rejected from the sample. For a handful of galaxies discrepancies were evident in multiple bands and the source of the error could not be identified nor remedied, so the relevant galaxy was rejected from the sample.

The residuals of the observed photometry and photometry synthesized from the spectra are summarized in Table 5. As some bands were used to normalize the spectra (i.e., $g$ band), we caution that some bands have small residuals by construction. The median and standard deviation of the offsets between the photometry and spectra are less than $10 \%$ for almost all of the bands.

After applying the correction for the $W 4$ filter curve error, the most significant residual is found in $5.8 \mu \mathrm{m}$ IRAC Channel-3
Table 5

Residuals between Photometry and Spectra

\begin{tabular}{|c|c|c|c|c|c|}
\hline \multirow[t]{2}{*}{ Filter } & \multirow{2}{*}{$\begin{array}{c}\lambda_{\text {eff }} \\
(\mu \mathrm{m})\end{array}$} & \multirow{2}{*}{$\begin{array}{l}\text { FWHM Bandwidth } \\
\qquad(\mu \mathrm{m})\end{array}$} & \multicolumn{2}{|c|}{$m_{\text {spec }}-m_{\text {phot }}$} & \multirow{2}{*}{$\begin{array}{c}\text { Number of } \\
\text { Galaxies }\end{array}$} \\
\hline & & & Median & Std. Dev. & \\
\hline FUV & 0.1531 & 0.0227 & 0.00 & 0.07 & 126 \\
\hline$U V W 2$ & 0.2026 & 0.0557 & 0.05 & 0.06 & 31 \\
\hline$U V M 2$ & 0.2238 & 0.0511 & -0.04 & 0.10 & 32 \\
\hline NUV & 0.2286 & 0.0795 & -0.01 & 0.07 & 126 \\
\hline$U V W 1$ & 0.2598 & 0.0681 & -0.04 & 0.08 & 27 \\
\hline$U$ & 0.3459 & 0.0557 & 0.08 & 0.14 & 5 \\
\hline$u$ & 0.3551 & 0.0582 & -0.01 & 0.06 & 129 \\
\hline$g$ & 0.4681 & 0.1262 & 0.00 & 0.00 & 129 \\
\hline$V$ & 0.5419 & 0.0730 & 0.09 & 0.10 & 3 \\
\hline$r$ & 0.6165 & 0.1149 & -0.02 & 0.02 & 129 \\
\hline$i$ & 0.7480 & 0.1238 & 0.01 & 0.03 & 129 \\
\hline$z$ & 0.8931 & 0.0994 & -0.03 & 0.03 & 129 \\
\hline$J$ & 1.232 & 0.215 & 0.03 & 0.05 & 129 \\
\hline$H$ & 1.644 & 0.263 & -0.00 & 0.06 & 129 \\
\hline$K_{S}$ & 2.159 & 0.279 & -0.00 & 0.06 & 128 \\
\hline$W 1$ & 3.357 & 0.793 & -0.02 & 0.03 & 128 \\
\hline [3.6] & 3.544 & 0.743 & 0.02 & 0.04 & 121 \\
\hline [4.5] & 4.487 & 1.010 & 0.01 & 0.08 & 122 \\
\hline$W 2$ & 4.606 & 1.106 & -0.06 & 0.07 & 128 \\
\hline [5.8] & 5.710 & 1.391 & 0.09 & 0.13 & 120 \\
\hline$[8.0]$ & 7.841 & 2.831 & 0.00 & 0.03 & 115 \\
\hline$W 3$ & 11.81 & 8.67 & -0.02 & 0.04 & 128 \\
\hline$P U I$ blue & 15.80 & 5.48 & -0.04 & 0.03 & 13 \\
\hline$W 4$ & 22.14 & 4.40 & 0.16 & 0.07 & 123 \\
\hline$W 4^{\prime}$ & 22.14 & 4.40 & -0.01 & 0.04 & 123 \\
\hline$P U I$ red & 22.32 & 7.30 & -0.10 & 0.05 & 12 \\
\hline [24] & 23.51 & 5.03 & 0.01 & 0.02 & 118 \\
\hline
\end{tabular}

photometry. In Figure 10 we plot the residuals as a function of galaxy color, with different symbols denoting different redshift ranges. While galaxies with Rayleigh-Jeans spectra show relatively modest errors, the photometry of star-forming galaxies is systematically brighter than the spectroscopy. As the presence of the residual depends on SED shape and is not seen in the other IRAC bands, it unlikely to result from errors in the scattered light corrections we have previously applied to the photometry. The $6.2 \mu \mathrm{m}$ PAH feature is near the red end of the $5.8 \mu \mathrm{m}$ filter curve, and should start moving out of the band toward the upper end of the redshift range. The systematic offset is removed if we shift the $5.8 \mu \mathrm{m}$ filter curve redward 


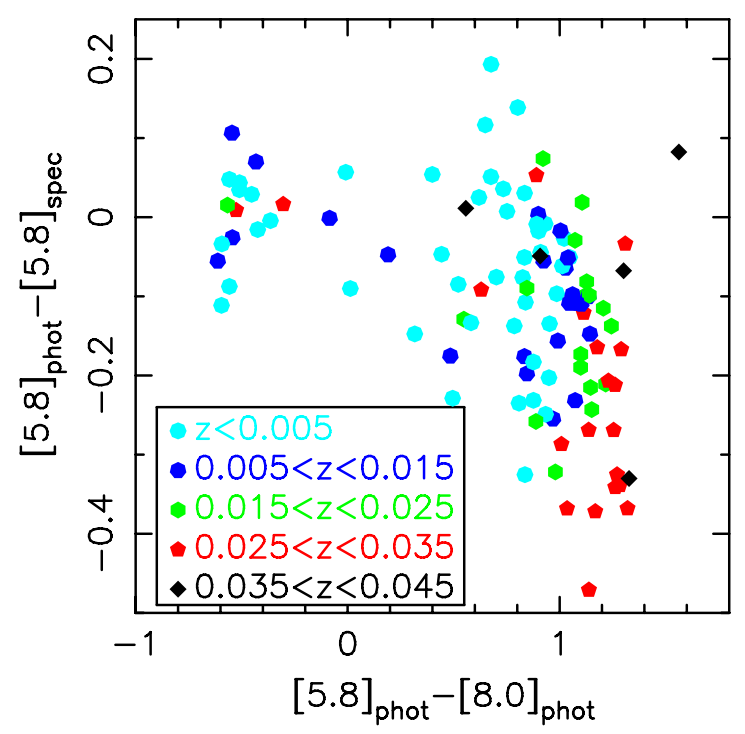

Figure 10. Offsets between the observed and synthesized $5.8 \mu \mathrm{m}$ photometry of galaxies. The $5.8 \mu \mathrm{m}$ photometry of star-forming galaxies is systematically brighter than the spectroscopy, but we have not (unambiguously) identified the cause of this offset.

(A color version of this figure is available in the online journal.)

by $1.5 \%$, although doing this may not be justified. The blue end of the IRS spectroscopy also overlaps the $5.8 \mu \mathrm{m}$ filter curve, and the spectra can have poor signal to noise shortward of the $6.2 \mu \mathrm{m}$ PAH feature. One could apply corrections to the IRS spectroscopy of star-forming galaxies, but doing so
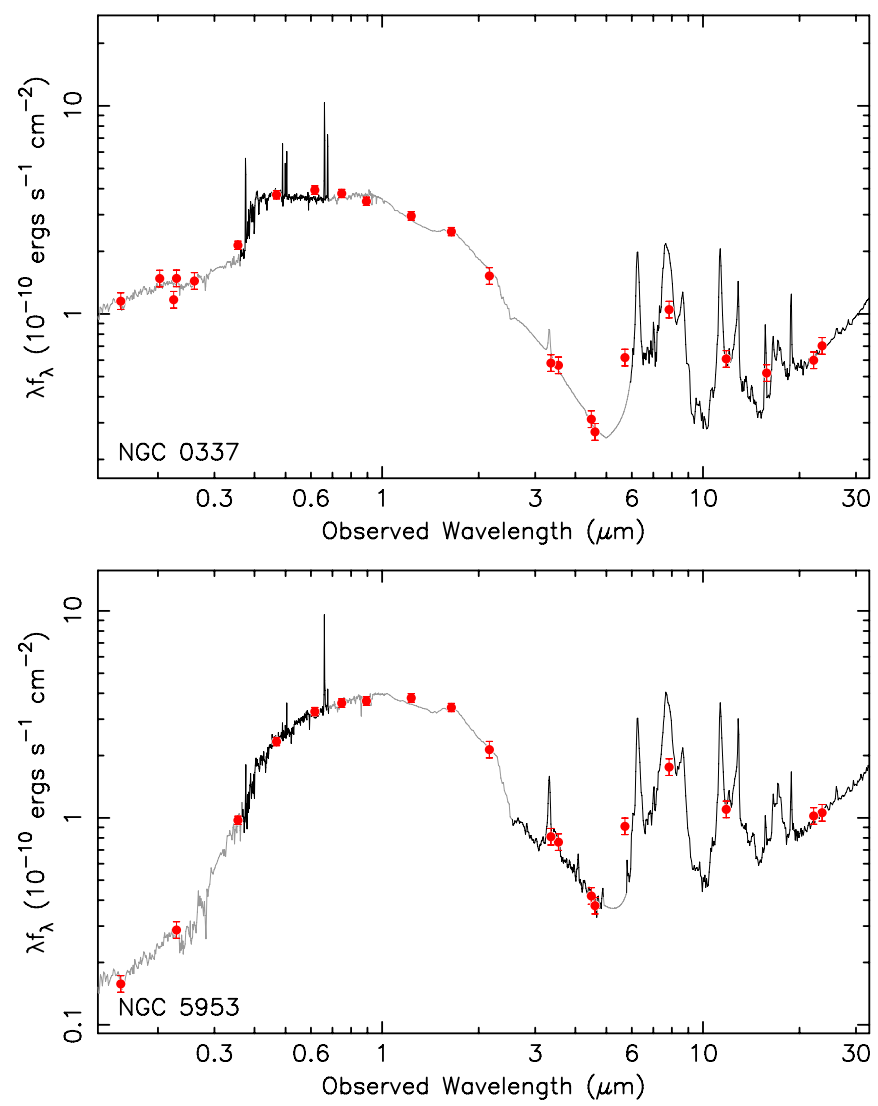

produces spectra that poorly match the Akari spectra at shorter wavelengths. We do not apply any corrections to the photometry or spectra $5.8 \mu \mathrm{m}$, but readers should be cautious of the spectra and photometry near this wavelength.

In Figure 11 we provide plots for four of the galaxies drawn from the illustrative subsample described in Appendix A. Spectra and images of all the galaxies in the sample are provided in Appendix B. Figure 11 shows good agreement between the photometry and spectroscopy, although we caution that some of this agreement is by construction (Section 4 compares the spectra with an independent set of photometry). Figure 11 also illustrates the diversity of the sample, including a passive earlytype galaxy (NGC 4125), a merging galaxy (NGC 5953), and a low metallicity dwarf galaxy (UGCA 219).

\section{COMPARISON WITH THE PRIOR LITERATURE}

Below we directly compare our SEDs (of particular galaxies) with those from the prior literature, and compare how well different template libraries reproduce the observed colors of galaxies. Systematic differences between our SEDs and those of previous template libraries are inevitable due to differences in methodology and data, with much of the data presented here not being available a decade ago. A key difference is the size of the aperture used to measure photometry and extract spectra, with some of the prior literature being restricted to galaxy nuclei (e.g., Kinney et al. 1996), which may not be representative of entire galaxies. We have deliberately produced spectra for individual galaxies, whereas the prior literature sometimes merged spectra from different galaxies to produce spectra spanning broad wavelength ranges. That said, these limitations and sources
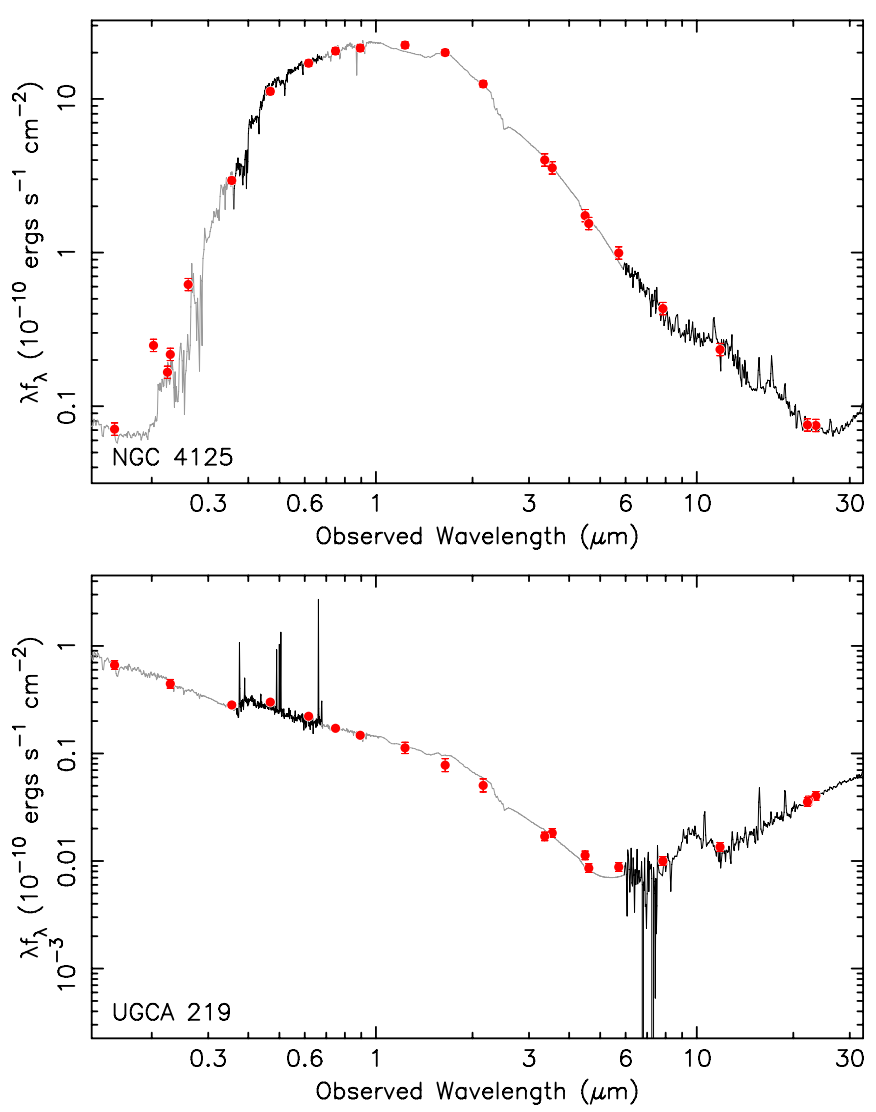

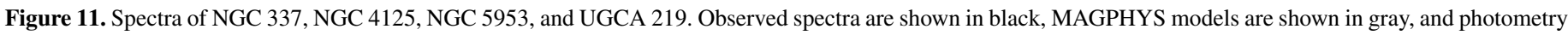
used to constrain and verify the spectra are shown with red dots. These galaxies are drawn from the illustrative subset of the sample described in Appendix A.

(A color version of this figure is available in the online journal.) 
Table 6

Factors Used to Rescale Spectra to Match Photometry

\begin{tabular}{|c|c|c|c|c|c|}
\hline \multirow[t]{2}{*}{ Name } & \multirow[t]{2}{*}{ Optical } & \multirow[t]{2}{*}{ Akari } & \multicolumn{3}{|c|}{ Spitzer IRS } \\
\hline & & & $8 \mu \mathrm{m}$ & $12 \mu \mathrm{m}$ & $20 \mu \mathrm{m}$ \\
\hline Arp $256 \mathrm{~N}$ & 1.09 & $\cdots$ & 2.27 & 1.90 & 0.91 \\
\hline Arp $256 \mathrm{~S}$ & 0.98 & 0.98 & 1.35 & 1.35 & 1.29 \\
\hline NGC 0337 & 1.02 & $\cdots$ & 1.03 & 1.07 & 1.06 \\
\hline CGCG 436-030 & 1.21 & 0.94 & 1.17 & 1.11 & 0.99 \\
\hline NGC 0474 & 1.30 & $\ldots$ & $\cdots$ & $\cdots$ & $\cdots$ \\
\hline NGC 0520 & 1.21 & 1.84 & 1.06 & 1.11 & 1.10 \\
\hline NGC 0584 & 1.20 & $\cdots$ & 2.22 & 2.12 & 1.38 \\
\hline NGC 0628 & 1.44 & $\ldots$ & 4.15 & 4.90 & 1.27 \\
\hline NGC 0660 & 1.78 & $\cdots$ & 3.60 & 3.04 & 1.35 \\
\hline III Zw 035 & 1.09 & 0.97 & 1.05 & 1.03 & 1.15 \\
\hline NGC 0695 & 1.15 & 1.22 & 2.45 & 2.36 & 1.27 \\
\hline NGC 0750 & 0.81 & $\cdots$ & $\cdots$ & $\cdots$ & $\cdots$ \\
\hline NGC 0855 & 1.27 & $\cdots$ & 1.37 & 1.52 & 1.10 \\
\hline NGC 1068 & 1.23 & 2.20 & 1.27 & 1.27 & 1.81 \\
\hline Arp 118 & 1.11 & $\cdots$ & 1.11 & 1.16 & 1.35 \\
\hline NGC 1144 & 1.12 & $\cdots$ & 1.10 & 1.15 & 1.33 \\
\hline NGC 1275 & 1.17 & 1.70 & 1.15 & 1.19 & 1.21 \\
\hline NGC 1614 & 1.01 & 1.10 & 2.13 & 2.17 & 1.50 \\
\hline NGC 2388 & 1.12 & 1.12 & 1.20 & 1.20 & 1.15 \\
\hline NGC 2403 & 1.36 & $\cdots$ & 2.15 & 2.40 & 1.70 \\
\hline NGC 2537 & 1.17 & $\cdots$ & $\cdots$ & $\cdots$ & $\cdots$ \\
\hline NGC 2623 & 0.98 & 1.19 & 1.08 & 1.08 & 1.10 \\
\hline IRAS 08572+3915 & 1.31 & 1.00 & 1.00 & 1.00 & 0.98 \\
\hline UGC 04881 & 1.11 & 1.41 & 2.75 & 2.55 & 1.72 \\
\hline NGC 2798 & 1.20 & $\cdots$ & 0.86 & 0.91 & 1.07 \\
\hline UGCA 166 & 1.21 & $\cdots$ & 1.94 & 1.94 & 1.10 \\
\hline UGC 05101 & 1.01 & 0.99 & 1.14 & 1.09 & 0.99 \\
\hline NGC 3049 & 1.25 & $\cdots$ & 1.09 & 1.15 & 1.11 \\
\hline NGC 3079 & 1.16 & $\cdots$ & 1.91 & 2.20 & 2.60 \\
\hline UGCA 208 & 1.08 & $\cdots$ & 1.24 & 1.02 & 1.17 \\
\hline NGC 3190 & 1.56 & $\cdots$ & 1.39 & 1.50 & 1.25 \\
\hline NGC 3198 & 1.24 & $\cdots$ & 1.47 & 1.29 & 1.39 \\
\hline NGC 3265 & 1.15 & $\ldots$ & 0.93 & 0.96 & 1.11 \\
\hline Mrk 33 & 1.17 & 4.15 & 0.96 & 1.03 & 1.24 \\
\hline NGC 3310 & 1.09 & $\cdots$ & 4.70 & 3.94 & 2.48 \\
\hline NGC 3351 & 1.13 & $\cdots$ & 1.15 & 1.14 & 1.18 \\
\hline NGC 3379 & $\ldots$ & $\ldots$ & $\ldots$ & $\ldots$ & $\ldots$ \\
\hline UGCA 219 & 1.12 & $\cdots$ & 1.66 & 1.68 & 1.19 \\
\hline NGC 3521 & 1.05 & $\cdots$ & 2.00 & 1.92 & 1.11 \\
\hline NGC 3627 & 1.31 & $\cdots$ & 3.31 & 3.41 & 1.16 \\
\hline IC 0691 & 1.20 & $\cdots$ & 1.50 & 1.57 & 1.07 \\
\hline NGC 3690 & 1.11 & 1.12 & 1.17 & 1.17 & 1.09 \\
\hline NGC 3773 & 1.12 & $\cdots$ & 1.01 & 1.06 & 1.27 \\
\hline Mrk 1450 & 1.14 & $\cdots$ & 1.28 & 1.06 & 0.92 \\
\hline UGC 06665 & 1.15 & $\cdots$ & 2.26 & 2.35 & 0.97 \\
\hline NGC 3870 & 1.06 & $\cdots$ & 2.14 & 2.02 & 0.89 \\
\hline UM 461 & 1.08 & $\cdots$ & 0.89 & 0.91 & 0.95 \\
\hline UGC 06850 & 1.16 & $\ldots$ & 2.62 & 1.83 & 1.17 \\
\hline NGC 3938 & 1.22 & $\ldots$ & 4.03 & 4.27 & 4.78 \\
\hline NGC 4088 & 1.03 & $\cdots$ & 3.25 & 3.40 & 2.19 \\
\hline NGC 4125 & 1.09 & $\cdots$ & 2.21 & 2.55 & 2.85 \\
\hline NGC 4138 & 1.05 & $\cdots$ & 1.27 & 1.23 & 1.13 \\
\hline NGC 4168 & $\cdots$ & $\cdots$ & $\cdots$ & $\cdots$ & $\cdots$ \\
\hline NGC 4194 & 1.05 & 2.85 & 1.47 & 1.52 & 1.10 \\
\hline Haro 06 & 1.04 & $\cdots$ & 0.88 & 1.00 & 2.09 \\
\hline NGC 4254 & 1.31 & $\cdots$ & 2.42 & 2.35 & 1.22 \\
\hline NGC 4321 & 1.28 & $\cdots$ & 2.12 & 2.40 & 1.21 \\
\hline NGC 4365 & $\cdots$ & $\cdots$ & $\cdots$ & $\cdots$ & $\cdots$ \\
\hline NGC 4387 & $\ldots$ & $\ldots$ & $\ldots$ & $\ldots$ & $\ldots$ \\
\hline NGC 4385 & 1.25 & $\cdots$ & 2.59 & 1.84 & 1.26 \\
\hline NGC 4450 & 1.51 & $\ldots$ & 2.32 & 2.26 & 2.17 \\
\hline NGC 4458 & $\cdots$ & $\cdots$ & $\cdots$ & $\cdots$ & $\cdots$ \\
\hline NGC 4473 & $\ldots$ & $\ldots$ & $\ldots$ & $\ldots$ & $\ldots$ \\
\hline
\end{tabular}

Table 6

(Continued)

\begin{tabular}{|c|c|c|c|c|c|}
\hline \multirow[t]{2}{*}{ Name } & \multirow[t]{2}{*}{ Optical } & \multirow[t]{2}{*}{ Akari } & \multicolumn{3}{|c|}{ Spitzer IRS } \\
\hline & & & $8 \mu \mathrm{m}$ & $12 \mu \mathrm{m}$ & $20 \mu \mathrm{m}$ \\
\hline NGC 4486 & $\ldots$ & $\ldots$ & $\ldots$ & $\ldots$ & $\ldots$ \\
\hline NGC 4536 & 1.54 & $\cdots$ & 1.22 & 1.27 & 1.11 \\
\hline NGC 4550 & $\cdots$ & $\cdots$ & $\cdots$ & $\cdots$ & $\cdots$ \\
\hline NGC 4551 & $\cdots$ & $\cdots$ & $\cdots$ & $\cdots$ & $\cdots$ \\
\hline NGC 4552 & 1.32 & $\cdots$ & 1.87 & 2.52 & 2.55 \\
\hline NGC 4559 & 1.46 & $\cdots$ & 3.38 & 3.60 & 1.22 \\
\hline NGC 4569 & 1.27 & $\ldots$ & 1.12 & 1.21 & 1.35 \\
\hline NGC 4579 & 1.12 & $\cdots$ & 1.54 & 1.49 & 1.25 \\
\hline NGC 4594 & 4.20 & $\cdots$ & 1.94 & 2.08 & 1.27 \\
\hline NGC 4625 & 1.17 & $\ldots$ & 1.74 & 1.86 & 2.09 \\
\hline NGC 4621 & $\cdots$ & $\cdots$ & $\cdots$ & $\cdots$ & $\cdots$ \\
\hline NGC 4631 & 1.04 & $\cdots$ & 3.37 & 3.66 & 1.18 \\
\hline NGC 4660 & $\ldots$ & $\ldots$ & $\ldots$ & $\ldots$ & $\ldots$ \\
\hline NGC 4670 & 1.14 & $\cdots$ & 2.32 & 1.88 & 1.18 \\
\hline NGC $4676 \mathrm{~A}$ & 0.60 & $\cdots$ & 1.01 & 1.05 & 1.29 \\
\hline NGC 4725 & 3.57 & $\cdots$ & 3.92 & 3.68 & 3.68 \\
\hline NGC 4826 & 1.32 & $\cdots$ & 2.13 & 2.20 & 2.20 \\
\hline NGC 4860 & $\ldots$ & $\cdots$ & $\cdots$ & $\cdots$ & $\cdots$ \\
\hline NGC 4889 & $\cdots$ & $\cdots$ & $\cdots$ & $\cdots$ & $\cdots$ \\
\hline IC 4051 & $\cdots$ & $\cdots$ & $\cdots$ & $\cdots$ & $\cdots$ \\
\hline NGC 4926 & $\ldots$ & $\cdots$ & $\cdots$ & $\cdots$ & $\cdots$ \\
\hline NGC 5033 & 1.43 & $\cdots$ & 1.86 & 1.83 & 1.16 \\
\hline IC 0860 & 1.09 & 1.04 & 1.19 & 1.03 & 0.91 \\
\hline UGC $08335 \mathrm{NW}$ & 1.34 & 1.94 & 1.37 & 1.37 & 1.27 \\
\hline UGC 08335 SE & 1.18 & 1.01 & 1.03 & 1.11 & 1.24 \\
\hline NGC 5055 & 1.26 & $\ldots$ & 3.65 & 4.06 & 1.32 \\
\hline IC 0883 & 0.77 & 1.02 & 1.15 & 1.17 & 1.02 \\
\hline NGC 5104 & 1.09 & 1.32 & 1.49 & 1.37 & 0.95 \\
\hline NGC 5194 & 1.16 & $\cdots$ & 3.05 & 3.32 & 1.18 \\
\hline NGC 5195 & 1.47 & $\cdots$ & 1.21 & 1.33 & 1.29 \\
\hline NGC 5256 & 1.12 & 1.06 & 0.81 & 0.81 & 1.48 \\
\hline NGC 5257 & 1.21 & $\ldots$ & 1.12 & 1.23 & 1.26 \\
\hline NGC 5258 & 1.18 & $\cdots$ & 1.15 & 1.25 & 1.30 \\
\hline UGC 08696 & 1.20 & 1.05 & 1.09 & 1.10 & 1.05 \\
\hline Mrk 1490 & 1.04 & 0.90 & 1.09 & 1.09 & 0.87 \\
\hline NGC 5653 & 1.12 & $\ldots$ & 1.07 & 1.10 & 1.11 \\
\hline Mrk 0475 & 1.03 & $\cdots$ & 1.89 & 1.34 & 0.97 \\
\hline NGC 5713 & 1.19 & $\cdots$ & 1.29 & 1.35 & 1.16 \\
\hline UGC $09618 \mathrm{~S}$ & 1.12 & $\cdots$ & 0.97 & 0.99 & 1.40 \\
\hline UGC 09618 & 0.94 & 1.18 & 1.02 & 1.03 & 1.20 \\
\hline UGC $09618 \mathrm{~N}$ & 1.14 & 0.96 & 1.07 & 1.08 & 1.24 \\
\hline NGC 5866 & 1.26 & $\ldots$ & 1.61 & 1.75 & 1.21 \\
\hline CGCG 049-057 & 1.03 & 0.99 & 1.25 & 1.25 & 1.01 \\
\hline NGC 5953 & 1.39 & 4.89 & 1.17 & 1.29 & 1.32 \\
\hline IC 4553 & 1.03 & 1.26 & 1.34 & 1.17 & 0.92 \\
\hline UGCA 410 & 1.17 & $\cdots$ & 1.07 & 1.07 & 0.92 \\
\hline NGC 5992 & 1.06 & 1.25 & 2.12 & 1.89 & 1.18 \\
\hline NGC 6052 & 1.11 & 3.90 & 1.11 & 1.17 & 1.33 \\
\hline NGC 6090 & 1.05 & 0.94 & 1.87 & 1.88 & 1.70 \\
\hline NGC 6240 & 1.15 & 1.18 & 1.01 & 1.06 & 1.09 \\
\hline IRAS $17208-0014$ & 1.22 & 1.17 & 1.24 & 1.07 & 1.04 \\
\hline II Zw 096 & 0.98 & 1.06 & 0.94 & 0.98 & 1.09 \\
\hline NGC 7331 & 1.38 & $\cdots$ & 4.99 & 5.22 & 1.20 \\
\hline UGC 12150 & 1.05 & 1.29 & 1.42 & 1.33 & 0.96 \\
\hline CGCG 453-062 & 1.75 & 1.12 & 1.81 & 1.66 & 1.15 \\
\hline IC 5298 & 1.09 & 1.11 & 1.29 & 1.12 & 0.97 \\
\hline NGC 7585 & 1.44 & $\ldots$ & $\ldots$ & $\ldots$ & $\cdots$ \\
\hline NGC 7591 & 1.04 & 1.44 & 1.36 & 1.34 & 1.29 \\
\hline NGC 7592 & 1.09 & 1.09 & 0.97 & 1.05 & 1.17 \\
\hline NGC 7673 & 1.16 & $\cdots$ & 1.45 & 1.45 & 1.06 \\
\hline NGC 7674 & 1.14 & 1.11 & 1.26 & 1.28 & 1.19 \\
\hline NGC 7679 & 1.13 & 1.17 & 1.86 & 1.66 & 1.10 \\
\hline Mrk 0930 & 1.18 & $\cdots$ & 2.65 & 2.01 & 1.00 \\
\hline NGC 7714 & 1.37 & 3.21 & 1.54 & 1.35 & 1.10 \\
\hline NGC 7771 & 1.29 & 3.98 & 1.81 & 1.78 & 1.22 \\
\hline Mrk 0331 & 1.12 & 1.05 & 1.29 & 1.30 & 1.00 \\
\hline
\end{tabular}




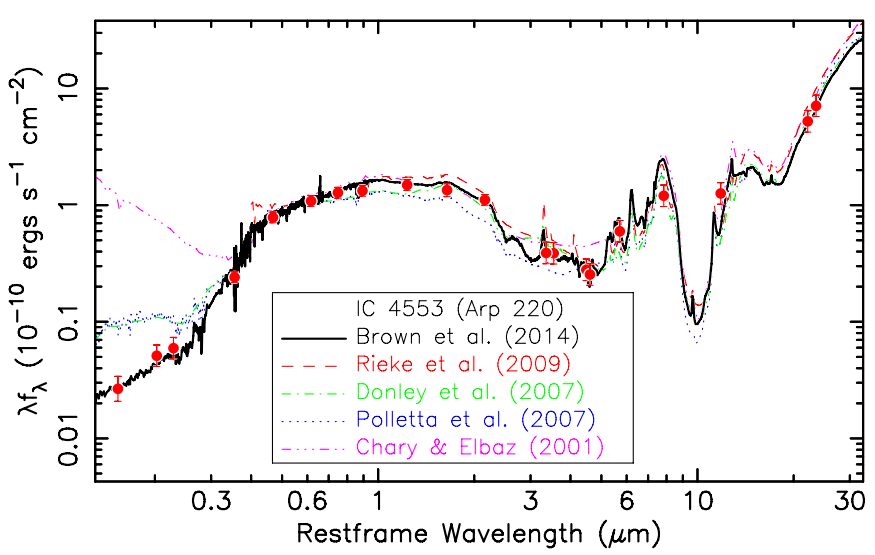

Figure 12. Our spectrum of IC 4553 (Arp 220) shown with IC 4553 template spectra taken from Chary \& Elbaz (2001), Donley et al. (2007), Polletta et al. (2007), and Rieke et al. (2009). To aid comparisons, the spectra have been normalized at $6000 \AA$ and our photometry is plotted with red dots. As noted by Chary \& Elbaz (2001), some LIRG and ULIRG SEDs from the literature were not constrained in the ultraviolet and consequently we see large differences between the templates at $2000 \AA$. The templates show significant differences across the entire wavelength range, and these differences could impact interpretations of galaxy properties derived from these templates.

(A color version of this figure is available in the online journal.)

of error were well understood and discussed by the relevant authors, who constructed the best SEDs possible with the data then available.

The biggest differences between our templates and the prior literature should be for LIRGs and ULIRGs, as these are difficult to model with stellar population synthesis models and are relatively faint in the ultraviolet. In Figure 12 we plot our spectrum of IC 4553 (Arp 220), along with other IC 4553 templates from the recent literature (Chary \& Elbaz 2001; Donley et al. 2007; Polletta et al. 2007; Rieke et al. 2009). While IC 4553 templates are frequently used to model the spectra of ULIRGs, the properties of this galaxy are unusual (e.g., Soifer et al. 1984) and ULIRG properties do display considerable diversity (e.g., Brandl et al. 2006; Howell et al. 2010; U et al. 2012).

It is immediately obvious from Figure 12 that the ultraviolet SEDs vary enormously, with the prior literature overestimating the $\sim 2000 \AA$ flux of IC 4553 by over $100 \%$. As with this work, the IC 4553 templates from the prior literature use combinations of spectra and model fits to photometry, using the data available at the time of publication. While it was reasonable for the prior literature to extend their IC 4553 SEDs into the ultraviolet (e.g., to approximate the observed optical colors of high redshift galaxies), previous IC 4553 SEDs had few (if any) constraints in ultraviolet (as noted by Chary \& Elbaz 2001), and consequently they systematically overestimated the ultraviolet flux of IC 4553. However, we caution that even with GALEX and Swift, we are constraining the ultraviolet SED of IC 4553 with just three photometric data points.

As can be seen in Figure 12, the shapes of the various IC 4553 (Arp 220) templates vary in the optical and mid-infrared too, with our spectrum featuring a relatively red $u-r$ color, strong PAH emission, and $3.1 \mu \mathrm{m}$ ice absorption feature. As all of the recent IC 4553 templates make use of (or have been updated with) Spitzer IRS spectra, the broad agreement beyond $5 \mu \mathrm{m}$ is not surprising. As IC 4553 template spectra are often used to interpret the observations of high redshift galaxies, it is plausible that errors in template spectra have led to erroneous conclusions. As we illustrate in Figure 13, the issues seen in IC 4553 templates are also seen in other LIRG SEDs. However, as
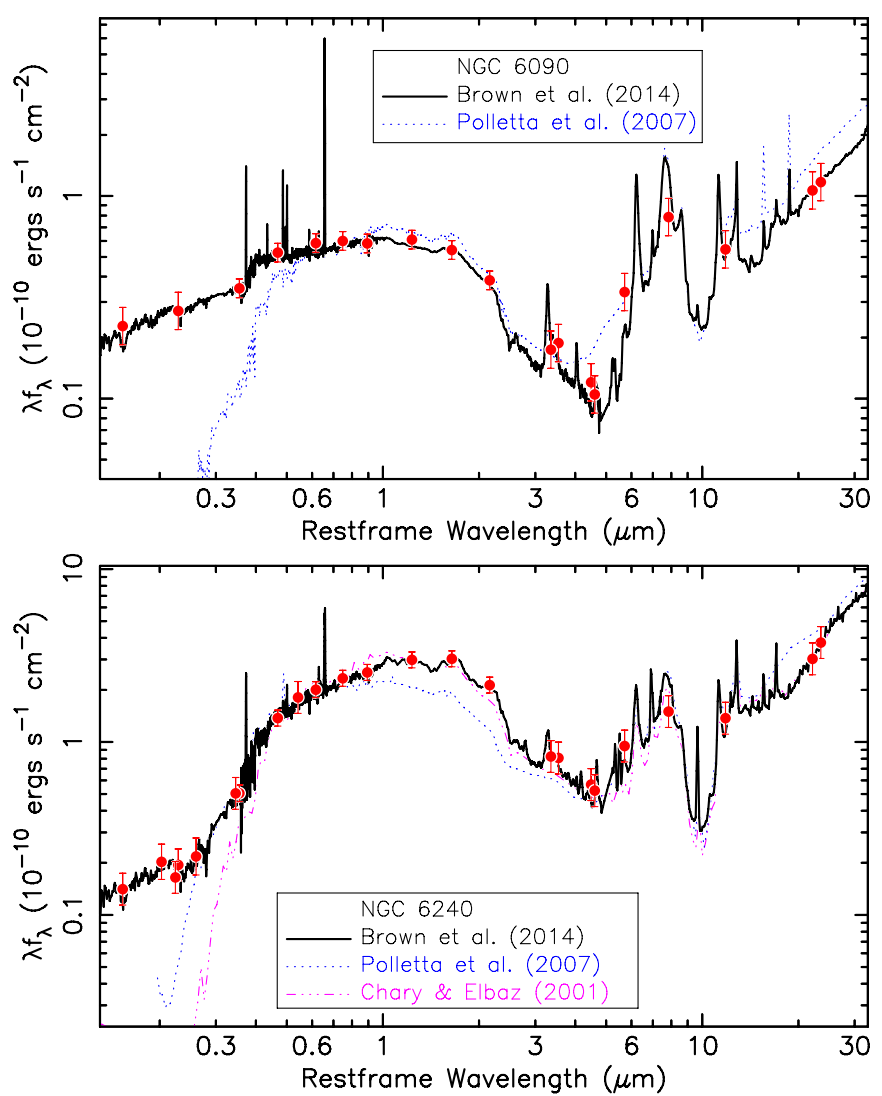

Figure 13. Our templates of NGC 6090 and NGC 6240 shown with the Polletta et al. (2007) templates for the same galaxies. The Polletta et al. (2007) templates for NGC 6090 and NGC 6240 use SEDs and photometry at wavelengths below $2 \mu \mathrm{m}$ that predate the advent of GALEX and SDSS imaging, and consequently we see significant errors at these wavelengths. The availability of well-calibrated imaging and matched-aperture photometry across a broad wavelength range (including redundant data) greatly simplifies the construction and validation of galaxy SEDs.

(A color version of this figure is available in the online journal.)

we discuss below, there is better agreement between different template libraries for galaxy types with little dust emission and no AGN content (e.g., ellipticals).

To compare our ensemble of templates with the prior literature, we generated synthetic colors and compared them to observed galaxy colors. We compare the performance of our templates with those from Coleman et al. (1980), Kinney et al. (1996) and Polletta et al. (2007), which have been used by a large variety of $K$-correction and photometric redshift codes over the past two decades (e.g., Fernández-Soto et al. 1999; Benítez 2000; Bolzonella et al. 2000; Ilbert et al. 2009). For this comparison we used optical and mid-infrared colors of $z \sim 0.3$ galaxies in the Boötes field (Jannuzi \& Dey 1999; Ashby et al. 2009). By changing redshift we avoid comparing our SEDs with photometry that has the same rest-frame wavelengths as the photometry used to constrain and verify the SEDs. The Boötes field optical imaging, taken from the National Optical Astronomy Observatory Deep Wide-Field Survey, also uses a different set of optical filters than the SDSS. The spectroscopic redshifts used for this comparison are taken from $I<20.4$ AGN and Galaxies Evolution Survey (Kochanek et al. 2012). The Boötes photometric catalogs are described in Brown et al. (2008) and we use a magnitude dependent aperture size to capture the vast majority of the galaxy light.

Figure 14 compares our templates, the Coleman et al. (1980) templates and the Kinney et al. (1996) templates with the 

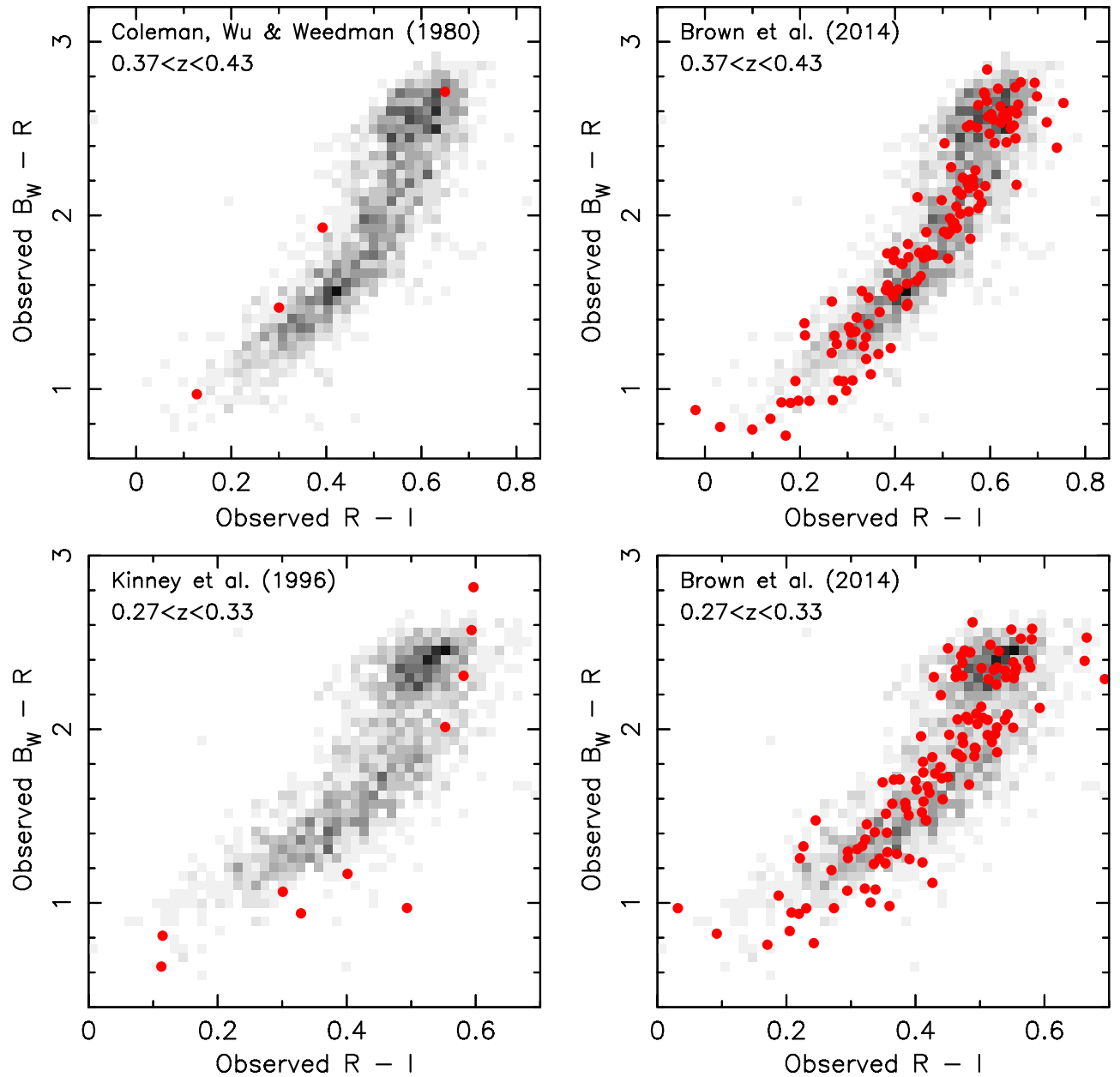

Figure 14. Comparison of the Coleman et al. (1980) and Kinney et al. (1996) templates (left panels) and our templates (right panels) with the observed optical colors of $z=0.4$ and $z=0.3$ galaxies in the Boötes field (grayscale). Both the Coleman et al. (1980) and Kinney et al. (1996) templates are slightly offset from the observed galaxy locus, whereas our templates follow the observed galaxy locus.

(A color version of this figure is available in the online journal.)
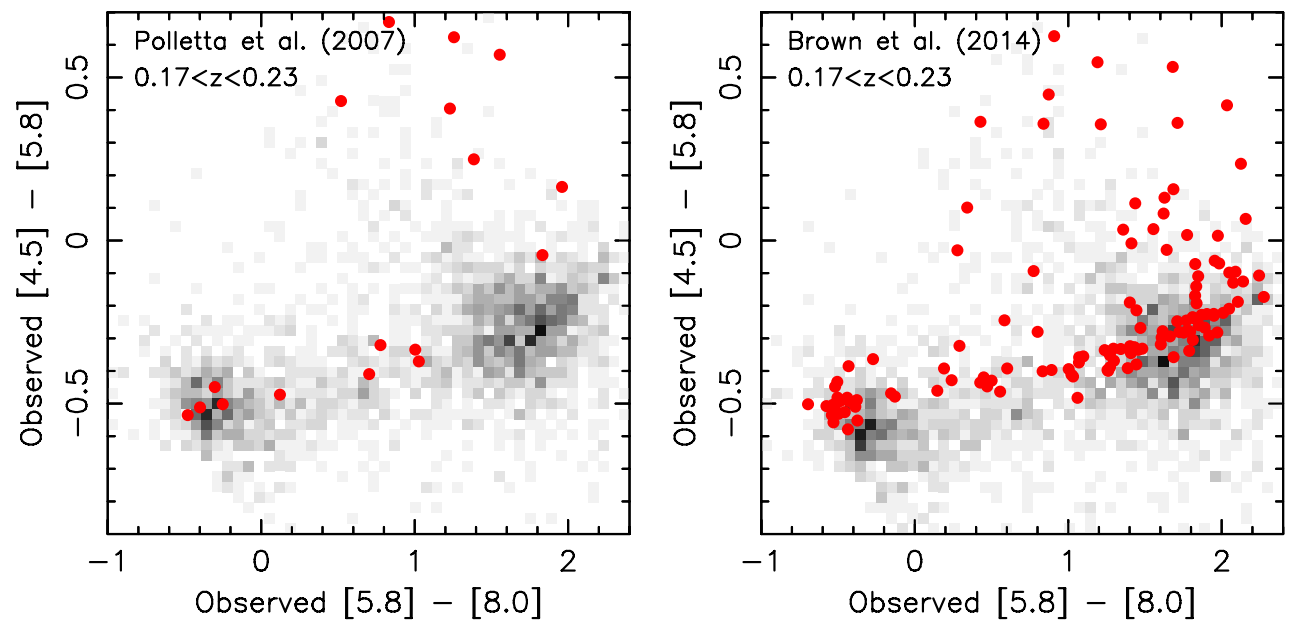

Figure 15. Comparison of the Polletta et al. (2007) templates (left panel) and our templates (right panel) with the observed mid-infrared colors of $z=0.2$ galaxies in the Boötes field (grayscale). The two loci in each panel correspond to galaxies with Rayleigh-Jeans spectra (left) and significant PAH emission (right). The Polletta et al. (2007) templates do not populate the locus of star-forming galaxies, whereas this region of the color-color diagram is well populated with our templates. It should be noted that our templates span a very broad range of galaxy types, and are not intended to mimic the frequency with which particular galaxies are observed. (A color version of this figure is available in the online journal.)

observed optical colors of $z=0.3$ and $z=0.4$ galaxies in Boötes. Both the Coleman et al. (1980) and Kinney et al. (1996) templates are systematically offset from the observed galaxy locus, and in some cases the offset corresponds to several tenths of a magnitude. Our templates broadly follow the observed galaxy loci in Figure 14. Some templates do fall off the galaxy locus, including blue emission line galaxies (which are generally fainter than $I=20.4$ at these redshifts) and LIRGs (that are 
relatively rare at $z \sim 0.3$ ). Some regions of the color-space that are populated by Kinney et al. (1996) templates are not populated by our templates. Given our large sample size and accurate photometry, we suspect this is due to errors in the Kinney et al. (1996) templates rather than incompleteness in our template library.

In Figure 15 we compare our templates and the Polletta et al. (2007) templates with the observed mid-infrared colors of $z \sim 0.2$ galaxies in Boötes. Galaxies with Rayleigh-Jeans SEDs and strong PAH emission form two distinct loci on the left and right of the plots. The PAH locus is not populated by the Polletta et al. (2007) templates, while multiple templates from our library populate the PAH locus. Our ability to populate the color-color diagram with templates is a consequence of the input photometry and spectroscopy, combined with our large sample size. However, even with 129 templates some regions of the color-color diagram are sparsely populated, including galaxies with mid-infrared colors that do not quite match Rayleigh-Jeans SEDs (e.g., galaxies similar to NGC 4450 and NGC 4725).

\section{SUMMARY}

We have constructed an atlas of 129 SEDs for nearby galaxies. $^{22}$ The atlas combines optical, Spitzer, and Akari spectra with MAGPHYS models, all of which have been normalized, constrained and verified with matched-aperture photometry. The redundancy of the 26 bands of matched-aperture photometry allows us to identify and mitigate systematic errors known to be present in the imaging data, including scattered light in IRAC images, coincidence losses in the Swift UVOT imaging and errors in the pre-launch WISE W4 filter curve. Comparison of the photometry and spectroscopy reveals that spectra used to calibrate star formation rate indicators systematically underestimate $\mathrm{H} \alpha$ fluxes by $10 \%$ or more. The SEDs are typically in good agreement with the photometry, with residuals being less than $10 \%$ in most cases.

Our atlas of SEDs spans a broad range of galaxy absolute magnitudes $\left(-14.7>M_{g}>-23.2\right)$, colors $(0.1<u-g<1.9)$, and types, including ellipticals, spirals, merging galaxies, blue compact dwarfs, and luminous infrared galaxies. Within individual object classes we see considerable diversity in our SEDs. For example, there is the well known diversity of ultraviolet SEDs for elliptical galaxies, and some elliptical galaxies have significant star formation and dust emission in the mid-infrared. Multi-wavelength photometry reveals the diversity of galaxy properties and shows that this diversity cannot be adequately modeled with a small number of galaxy templates.

Our SEDs differ significantly from those in the prior literature, particularly for LIRGs. Relative to the prior literature, our SEDs provide a better match to the observed optical and mid-infrared colors of $z<0.5$ galaxies. Our atlas will provide improved $K$-corrections, photometric redshifts, and local analogs of distant galaxies than existing template libraries. However, significant improvements can still be made including extending the atlas to longer wavelengths and filling gaps in the spectral coverage in the ultraviolet and near-infrared.

We thank Vianney Lebouteiller for responding to our queries about and making improvements to the Cornell Atlas of Spitzer/IRS Sources (CASSIS Lebouteiller et al. 2011).

\footnotetext{
22 The 129 SEDs and associated images are available via
} http://dx.doi.org/10.5072/03/529D3551F0117.
We thank Michelle Cluver for using and providing feedback on a preliminary version of the templates (Cluver et al. 2013, 2014). We also thank Richard Beare, Mark Brodwin, Vassilis Charmandaris, Charlie Conroy, Simon Driver, Peppo Gavazzi, Will Hartley, Erik Hoversten, Hanae Inami, Rob Kennicutt, and Lauranne Lanz for their assistance and feedback as the spectral templates were being developed. Michael Brown acknowledges financial support from The Australian Research Council (FT100100280), the Monash Research Accelerator Program (MRA), and the International Science Linkages Program. Part of the research for this paper was conducted during a visit to the Aspen Center for Physics in 2012 June. Masa Imanishi is supported by Grants-in-Aid for Scientific Research (No. 22012006).

Swift UVOT was designed and built in collaboration between MSSL, PSU, SwRI, Swales Aerospace, and GSFC, and was launched by NASA. GALEX is a NASA Small Explorer, launched in 2003 April. We gratefully acknowledge NASA's support for construction, operation, and science analysis for the GALEX mission, developed in cooperation with the Centre National d'Etudes Spatiales of France and the Korean Ministry of Science and Technology.

Funding for SDSS-III has been provided by the Alfred P. Sloan Foundation, the Participating Institutions, the National Science Foundation, and the U.S. Department of Energy Office of Science. The SDSS-III Web site is http://www.sdss3.org/. SDSS-III is managed by the Astrophysical Research Consortium for the Participating Institutions of the SDSS-III Collaboration including the University of Arizona, the Brazilian Participation Group, Brookhaven National Laboratory, University of Cambridge, University of Florida, the French Participation Group, the German Participation Group, the Instituto de Astrofisica de Canarias, the Michigan State/Notre Dame/ JINA Participation Group, Johns Hopkins University, Lawrence Berkeley National Laboratory, Max Planck Institute for Astrophysics, New Mexico State University, New York University, Ohio State University, Pennsylvania State University, University of Portsmouth, Princeton University, the Spanish Participation Group, University of Tokyo, University of Utah, Vanderbilt University, University of Virginia, University of Washington, and Yale University. The NASA-Sloan Atlas was created by Michael Blanton, with extensive help and testing from Eyal Kazin, Guangtun Zhu, Adrian Price-Whelan, John Moustakas, Demitri Muna, Renbin Yan, and Benjamin Weaver. Funding for the NASA-Sloan Atlas has been provided by the NASA Astrophysics Data Analysis Program (08-ADP08-0072) and the NSF (AST-1211644).

This research is based in part on observations taken with telescopes of the National Optical Astronomy Observatory, which is operated by the Association of Universities for Research in Astronomy (AURA) under cooperative agreement with the National Science Foundation. This publication makes use of data products from the Two Micron All Sky Survey, which is a joint project of the University of Massachusetts and the Infrared Processing and Analysis Center/California Institute of Technology, funded by the National Aeronautics and Space Administration and the National Science Foundation. This research is based in part on observations with Akari, a JAXA project with the participation of ESA.

This work is based in part on observations made with the Spitzer Space Telescope, obtained from the NASA/IPAC Infrared Science Archive, both of which are operated by the Jet Propulsion Laboratory, California Institute of Technology 


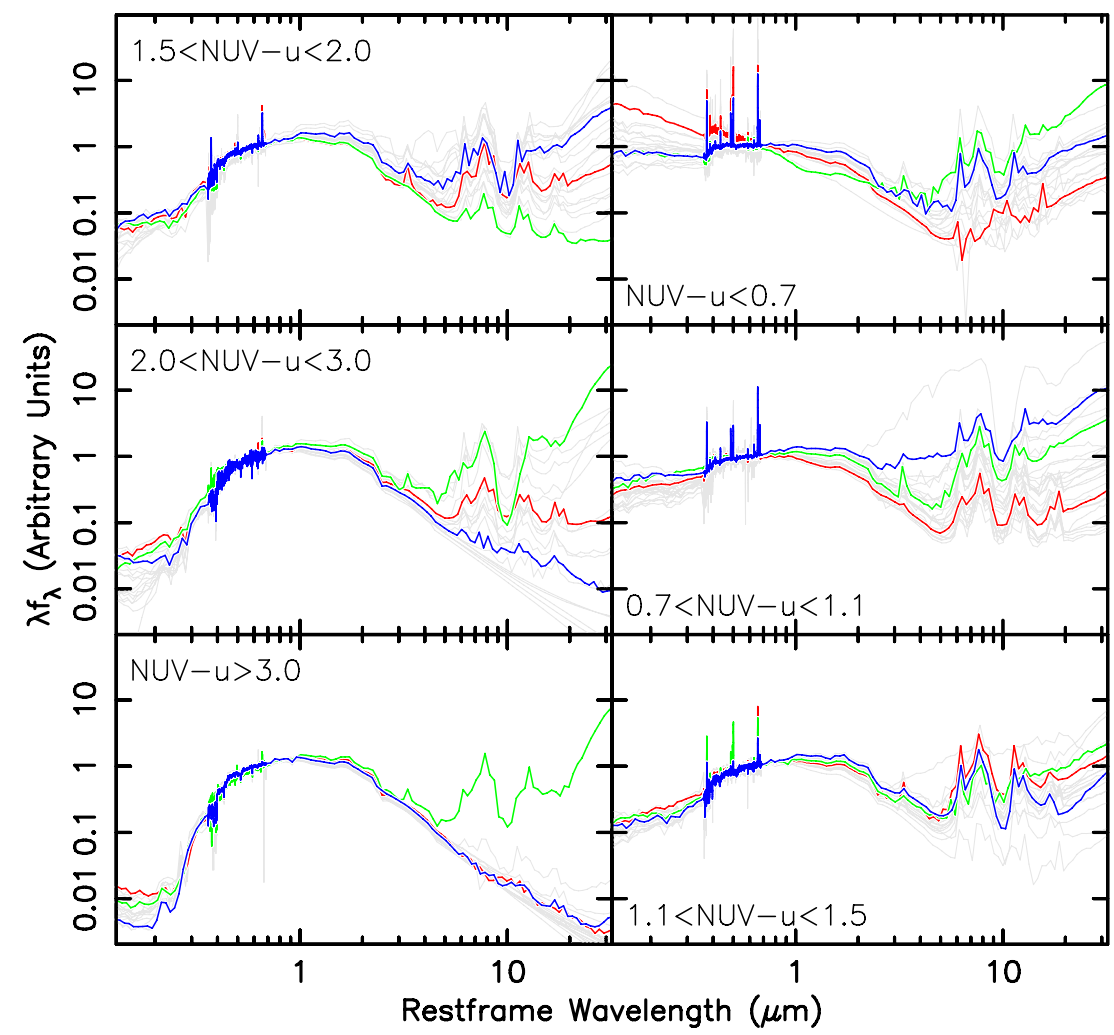

Figure 16. Spectra of the 18 subsample galaxies. Each panel shows a different range of NUV $-u$ color, and for comparison the spectra of main sample galaxies are shown in gray. The subsample spans much of the color range of the full sample and includes a diversity of galaxy types, including ellipticals, spirals, blue compact dwarfs, mergers, LIRGs, and starbursts.

(A color version of this figure is available in the online journal.)
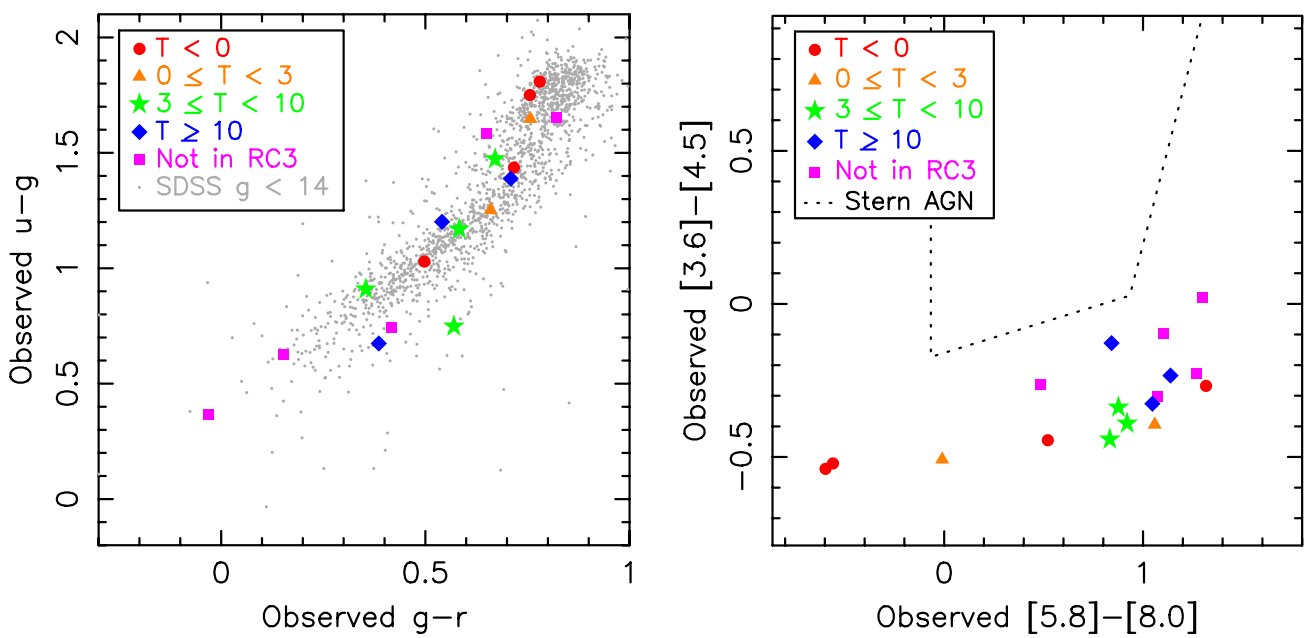

Figure 17. Observed optical and Spitzer IRAC colors of the 18 subsample galaxies listed in Table 7. The subsample is intended to span the observed range of galaxy types and colors seen in the nearby universe and is not intended to be representative of nearby galaxy populations.

(A color version of this figure is available in the online journal.)

under a contract with the National Aeronautics and Space Administration. The Cornell Atlas of Spitzer/IRS Sources (CASSIS) is a product of the Infrared Science Center at Cornell University, supported by NASA and JPL. This publication makes use of data products from the Wide-field Infrared Survey Explorer, which is a joint project of the University of California, Los Angeles, and the Jet Propulsion Laboratory/California Institute of Technology, funded by the National Aeronautics and Space Administration.

Facilities: Akari (IRC), Bok (Boller \& Chivens spectrograph), CTIO:1.5m (R-C spectrograph), CTIO:2MASS,
FLWO:2MASS, GALEX, Mayall (MOSAIC-1 wide-field camera), MMT (Hectospec), Sloan, Spitzer (IRAC, IRS, MIPS), Swift, WISE

\section{APPENDIX A}

\section{AN ILLUSTRATIVE SUBSAMPLE}

In Figure 16 and Table 7 we present an illustrative subsample of 18 galaxy spectra. We present spectra of individual galaxies rather than averaging spectra, as averaging can produce spectra that do not match those of real galaxies. As we are providing 

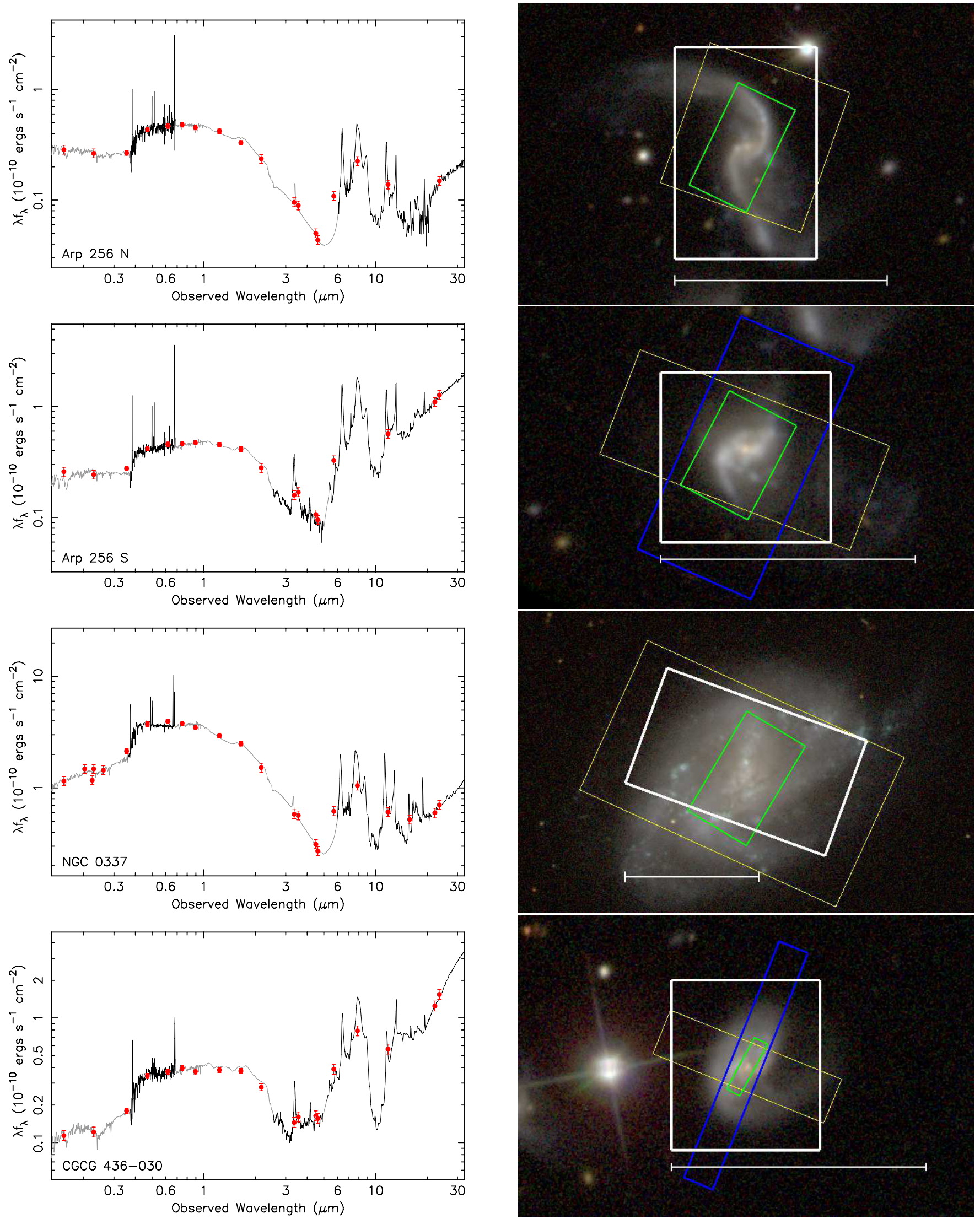

Figure 18. Galaxy SEDs from the ultraviolet to the mid-infrared. In the left panel the observed and model spectra are shown in black and gray respectively, while the photometry used to constrain and verify the spectra is shown with red dots. In the right panel we plot (where applicable) the photometric aperture (thick white rectangle), the Akari extraction aperture (blue rectangle), the Spitzer SL extraction aperture (green rectangle) and the Spitzer LL extraction aperture (yellow rectangle). For galaxies with Spitzer stare mode spectra, we show a region corresponding to a quarter of the slit length. For scale, the horizontal bar denotes 1'.

(A color version and complete figure set (258 images) for this figure are available in the online journal.) 
Table 7

Basic Properties of the Subsample

\begin{tabular}{|c|c|c|c|c|c|c|}
\hline Name & Morphology & T-type & $g$ & $M_{\mathrm{NUV}}-M_{u}$ & $M_{[8.0]}-M_{[24]}$ & Notes \\
\hline NGC 0337 & SBd & 7.0 & 12.36 & 0.96 & 0.79 & \\
\hline NGC 0695 & S0 & -2.0 & 13.90 & 1.16 & 0.72 & Interacting galaxy \\
\hline NGC 3079 & $\mathrm{SB}(\mathrm{s}) \mathrm{c}$ & 7.0 & 11.07 & 1.31 & 0.27 & Seyfert 2 \\
\hline Mrk 33 & Im pec & 10.0 & 13.36 & 0.53 & 2.09 & Wolf-Rayet galaxy \\
\hline UGCA 219 & $\mathrm{Sc}$ & $\cdots$ & 14.78 & 0.01 & 2.76 & Blue compact dwarf \\
\hline NGC 3521 & $\mathrm{SABbc}$ & 4.0 & 10.38 & 2.02 & 0.05 & \\
\hline NGC 3690 & Pec & 9.0 & 12.07 & 0.72 & 2.19 & Galaxy merger and Wolf-Rayet galaxy \\
\hline NGC 4125 & E6 pec & -5.0 & 10.89 & 3.44 & -0.70 & Elliptical galaxy without UV upturn \\
\hline NGC 4138 & $\mathrm{SA}(\mathrm{r}) 0$ & -1.0 & 11.86 & 1.83 & 0.04 & \\
\hline NGC 4552 & $\mathrm{E}$ & -5.0 & 10.94 & 3.01 & -0.74 & Elliptical galaxy with UV upturn \\
\hline NGC 4725 & SABab pec & 2.0 & 11.08 & 2.52 & -0.20 & Seyfert 2 \\
\hline NGC 5256 & Pec & 99.0 & 13.82 & 1.35 & 2.14 & Galaxy merger \\
\hline CGCG 049-057 & Irr & $\cdots$ & 15.16 & 3.17 & 2.32 & LIRG \\
\hline NGC 5953 & $\mathrm{Sa}$ & 1.0 & 12.65 & 1.89 & 0.67 & \\
\hline IC 4553 & Pec & $\cdots$ & 13.85 & 2.35 & 3.27 & ULIRG Arp 220 \\
\hline NGC 6090 & Pec & $\cdots$ & 14.20 & 0.82 & 1.76 & Galaxy merger and LIRG \\
\hline NGC 6240 & Pec & 90.0 & 13.45 & 1.69 & 2.34 & \\
\hline II Zw 096 & Pec & $\cdots$ & 14.29 & 0.50 & 2.91 & Galaxy merger and starburst \\
\hline
\end{tabular}

spectra of individual galaxies, it is also possible for others to add spectra to these templates as new data becomes available. The subsample was selected to span a broad range of NUV $-u$ color, and for each NUV $-u$ color bin shown in Figure 16 we chose three galaxies which have differing mid-infrared SEDs. Where possible, we selected galaxies that had Akari spectra, particularly when galaxies had strong mid-infrared emission and were thus likely to have strong PAH emission and the $3.1 \mu \mathrm{m}$ ice absorption feature. The observed colors of the subsample galaxies are plotted in Figure 17. We caution that our subsample does not include some of the most extreme galaxies from the overall sample (e.g., IRAS 08572+3915, UGC 5101) and none of the subsample galaxies meet the Stern et al. (2005) AGN selection criterion. With some exceptions, including LIRGs, the optical colors of the subsample galaxies lie close to the locus of NASA-Sloan Atlas galaxies.

\section{APPENDIX B}

\section{PRESENTATION OF THE SPECTRA}

In Figure 18 we present the spectra and gri images of the sample galaxies (the full figure is available online). Overlaid on the gri images are the photometric apertures and the apertures used to extract the Akari and Spitzer spectra (defined in Table 2). When Spitzer stare mode spectra were used, the length of the slit used for the extraction varies with wavelength, so we plot a quarter of the slit width in these instances. The plots of the individual galaxy spectra include the dust corrected photometry used to constrain and verify the spectroscopy (also see Table 3), including corrections for Swift UVOT coincidence losses and errors in the WISE W4 filter curve.

\section{REFERENCES}

Aihara, H., Allende Prieto, C., An, D., et al. 2011, ApJS, 193, 29

Armus, L., Mazzarella, J. M., Evans, A. S., et al. 2009, PASP, 121, 559

Ashby, M. L. N., Stern, D., Brodwin, M., et al. 2009, ApJ, 701, 428

Assef, R. J., Kochanek, C. S., Brodwin, M., et al. 2008, ApJ, 676, 286

Atek, H., Siana, B., Scarlata, C., et al. 2011, ApJ, 743, 121

Benítez, N. 2000, ApJ, 536, 571

Bertin, E., Mellier, Y., Radovich, M., et al. 2002, in ASP Conf. Ser. 281, Astronomical Data Analysis Software and Systems XI, ed. D. A. Bohlender, D. Durand, \& T. H. Handley (San Francisco, CA: ASP), 228
Blanton, M. R., Kazin, E., Muna, D., Weaver, B. A., \& Price-Whelan, A. 2011, AJ, 142, 31

Blanton, M. R., \& Roweis, S. 2007, AJ, 133, 734

Bolzonella, M., Miralles, J.-M., \& Pelló, R. 2000, A\&A, 363, 476

Brandl, B. R., Bernard-Salas, J., Spoon, H. W. W., et al. 2006, ApJ, 653, 1129

Breeveld, A. A., Curran, P. A., Hoversten, E. A., et al. 2010, MNRAS, 406, 1687

Brown, M. J. I., Zheng, Z., White, M., et al. 2008, ApJ, 682, 937

Bruzual, G., \& Charlot, S. 2003, MNRAS, 344, 1000

Cardamone, C. N., Urry, C. M., Schawinski, K., et al. 2010, ApJL, 721, L38

Charlot, S., \& Longhetti, M. 2001, MNRAS, 323, 887

Chary, R., \& Elbaz, D. 2001, ApJ, 556, 562

Cluver, M. E., Appleton, P. N., Ogle, P., et al. 2013, ApJ, 765, 93

Cluver, M. E., Jarrett, T. H., Hopkins, A. M., et al. 2014, ApJ, 782, 90

Code, A. D., \& Welch, G. A. 1979, ApJ, 228, 95

Coleman, G. D., Wu, C.-C., \& Weedman, D. W. 1980, ApJS, 43, 393

Connolly, A. J., Csabai, I., Szalay, A. S., et al. 1995, AJ, 110, 2655

da Cunha, E., Charlot, S., \& Elbaz, D. 2008, MNRAS, 388, 1595

de Vaucouleurs, G., de Vaucouleurs, A., Corwin, H. G., Jr., et al. 1991 , Third Reference Catalogue of Bright Galaxies. Volume I: Explanations and References. Volume II: Data for Galaxies between $0^{h}$ and $12^{h}$. Volume III: Data for Galaxies between $12^{h}$ and $24^{h}$ (New York: Springer)

Donas, J., Deharveng, J.-M., Laget, M., Milliard, B., \& Huguenin, D. 1987, A\&A, 180, 12

Donas, J., Deharveng, J.-M., Rich, R. M., et al. 2007, ApJS, 173, 597

Donley, J. L., Rieke, G. H., Pérez-González, P. G., Rigby, J. R., \& AlonsoHerrero, A. 2007, ApJ, 660, 167

Engelbracht, C. W., Rieke, G. H., Gordon, K. D., et al. 2008, ApJ, 678, 804

Fazio, G. G., Hora, J. L., Allen, L. E., et al. 2004, ApJS, 154, 10

Fernández-Soto, A., Lanzetta, K. M., \& Yahil, A. 1999, ApJ, 513, 34

Firth, A. E., Lahav, O., \& Somerville, R. S. 2003, MNRAS, 339, 1195

Fitzpatrick, E. L. 1999, PASP, 111, 63

Gavazzi, G., Zaccardo, A., Sanvito, G., Boselli, A., \& Bonfanti, C. 2004, A\&A, 417,499

Hogg, D. W., Baldry, I. K., Blanton, M. R., \& Eisenstein, D. J. 2002, arXiv:astro-ph/0210394

Houck, J. R., Roellig, T. L., van Cleve, J., et al. 2004, ApJS, 154, 18

Hoversten, E. A., Gronwall, C., Vanden Berk, D. E., et al. 2011, AJ, 141, 205

Howell, J. H., Armus, L., Mazzarella, J. M., et al. 2010, ApJ, 715, 572

Howell, J. H., Mazzarella, J. M., Chan, B. H. P., et al. 2007, AJ, 134, 2086

Ilbert, O., Capak, P., Salvato, M., et al. 2009, ApJ, 690, 1236

Imanishi, M., Nakagawa, T., Shirahata, M., Ohyama, Y., \& Onaka, T. 2010, ApJ, 721,1233

Inami, H., Armus, L., Charmandaris, V., et al. 2013, ApJ, 777, 156

Jannuzi, B. T., \& Dey, A. 1999, in ASP Conf. Ser. 191, Photometric Redshifts and the Detection of High Redshift Galaxies, ed. R. Weymann, L. StorrieLombardi, M. Sawicki, \& R. Brunner (San Francisco, CA: ASP), 111

Jarrett, T. H., Chester, T., Cutri, R., Schneider, S. E., \& Huchra, J. P. 2003, AJ, 125,525

Jarrett, T. H., Cohen, M., Masci, F., et al. 2011, ApJ, 735, 112 
Jarrett, T. H., Masci, F., Tsai, C. W., et al. 2012, AJ, 144, 68

Jarrett, T. H., Masci, F., Tsai, C. W., et al. 2013, AJ, 145, 6

Kennicutt, R. C., Jr. 1992, ApJS, 79, 255

Kennicutt, R. C., Jr., Armus, L., Bendo, G., et al. 2003, PASP, 115, 928

Kennicutt, R. C., Jr., Hao, C.-N., Calzetti, D., et al. 2009, ApJ, 703, 1672

Kessler, M. F., Steinz, J. A., Anderegg, M. E., et al. 1996, A\&A, 315, L27

Kinney, A. L., Bohlin, R. C., Calzetti, D., Panagia, N., \& Wyse, R. F. G. 1993, ApJS, 86, 5

Kinney, A. L., Calzetti, D., Bohlin, R. C., et al. 1996, ApJ, 467, 38

Kochanek, C. S., Eisenstein, D. J., Cool, R. J., et al. 2012, ApJS, 200, 8

Lebouteiller, V., Barry, D. J., Spoon, H. W. W., et al. 2011, ApJS, 196, 8

Maraston, C. 2005, MNRAS, 362, 799

Mason, K. O., Breeveld, A., Much, R., et al. 2001, A\&A, 365, L36

Morrissey, P., Conrow, T., Barlow, T. A., et al. 2007, ApJS, 173, 682

Moustakas, J., \& Kennicutt, R. C., Jr. 2006, ApJS, 164, 81

Moustakas, J., Kennicutt, R. C., Jr., \& Tremonti, C. A. 2006, ApJ, 642, 775

Moustakas, J., Kennicutt, R. C., Jr., Tremonti, C. A., et al. 2010, ApJS, 190, 233

Murakami, H., Baba, H., Barthel, P., et al. 2007, PASJ, 59, 369

Ohyama, Y., Onaka, T., Matsuhara, H., et al. 2007, PASJ, 59, 411

Oke, J. B., \& Sandage, A. 1968, ApJ, 154, 21

Onaka, T., Matsuhara, H., Wada, T., et al. 2007, PASJ, 59, 401

Pacifici, C., Charlot, S., Blaizot, J., \& Brinchmann, J. 2012, MNRAS, 421, 2002

Peek, J. E. G. 2013, arXiv:1131.2941

Peek, J. E. G., \& Schiminovich, D. 2013, ApJ, 771, 68

Pence, W. 1976, ApJ, 203, 39
Pérez-Montero, E., Hägele, G. F., Contini, T., \& Díaz, Á. I. 2007, MNRAS, 381,125

Petric, A. O., Armus, L., Howell, J., et al. 2011, ApJ, 730, 28

Pforr, J., Maraston, C., \& Tonini, C. 2012, MNRAS, 422, 3285

Planck Collaboration, Ade, P. A. R., Aghanim, N., et al. 2011, A\&A, 536, A19

Planck Collaboration, Ade, P. A. R., Aghanim, N., et al. 2013, arXiv:1303.5062

Polletta, M., Tajer, M., Maraschi, L., et al. 2007, ApJ, 663, 81

Rieke, G. H., Alonso-Herrero, A., Weiner, B. J., et al. 2009, ApJ, 692, 556

Rieke, G. H., Young, E. T., Engelbracht, C. W., et al. 2004, ApJS, 154, 25

Roming, P. W. A., Kennedy, T. E., Mason, K. O., et al. 2005, SSRv, 120, 95

Schaerer, D., \& de Barros, S. 2009, A\&A, 502, 423

Silva, L., Granato, G. L., Bressan, A., \& Danese, L. 1998, ApJ, 509, 103

Skrutskie, M. F., Cutri, R. M., Stiening, R., et al. 2006, AJ, 131, 1163

Smith, J. D. T., Armus, L., Dale, D. A., et al. 2007a, PASP, 119, 1133

Smith, J. D. T., Draine, B. T., Dale, D. A., et al. 2007b, ApJ, 656, 770

Soifer, B. T., Neugebauer, G., Helou, G., et al. 1984, ApJL, 283, L1

Stern, D., Eisenhardt, P., Gorjian, V., et al. 2005, ApJ, 631, 163

Stierwalt, S., Armus, L., Surace, J. A., et al. 2013, ApJS, 206, 1

Tinsley, B. M. 1968, ApJ, 151, 547

U, V., Sanders, D. B., Mazzarella, J. M., et al. 2012, ApJS, 203, 9

Vaduvescu, O., McCall, M. L., Richer, M. G., \& Fingerhut, R. L. 2005, AJ, 130,1593

Vega, O., Clemens, M. S., Bressan, A., et al. 2008, A\&A, 484, 631

Werner, M. W., Roellig, T. L., Low, F. J., et al. 2004, ApJS, 154, 1

Wright, E. L., Eisenhardt, P. R. M., Mainzer, A. K., et al. 2010, AJ, 140, 1868

Wu, Y., Charmandaris, V., Hao, L., et al. 2006, ApJ, 639, 157 